\title{
Hepatobiliary and pancreatic imaging in children-techniques and an overview of non-neoplastic disease entities
}

\author{
Rutger A. J. Nievelstein • Simon G. F. Robben • \\ Johan G. Blickman
}

Received: 24 May 2010 /Revised: 10 August 2010/Accepted: 30 August 2010 /Published online: 22 October 2010

(C) The Author(s) 2010. This article is published with open access at Springerlink.com

\begin{abstract}
Imaging plays a major role in the diagnostic work-up of children with hepatobiliary or pancreatic diseases. It consists mainly of US, CT and MRI, with US and MRI being the preferred imaging modalities because of the lack of ionizing radiation. In this review the technique of US, CT and MRI in children will be addressed, followed by a comprehensive overview of the imaging characteristics of several hepatobiliary and pancreatic disease entities most common in the paediatric age group.
\end{abstract}

Keywords Liver - Pancreas · Bile duct · US · CT · MRI . Child

\section{Introduction}

Imaging of the paediatric hepatobiliary and pancreatic systems consists mainly of US, CT and MRI. All these techniques have undergone major technical developments in the last decades, leading to a substantial increase in their diagnostic applications and accuracy in children. Today, US and MRI have become the preferred imaging modalities

R. A. J. Nievelstein $(\bowtie)$

Department of Radiology E01.132,

University Medical Center Utrecht,

Wilhelmina Children's Hospital,

P.O. Box 85500, 3508 GA Utrecht, The Netherlands

e-mail: R.A.J.Nievelstein@umcutrecht.nl

\section{S. G. F. Robben}

Department of Radiology, University Medical Center Maastricht, Maastricht, The Netherlands

J. G. Blickman

Department of Imaging Sciences, Golisano Children's Hospital,

Rochester, NY, USA over $\mathrm{CT}$ and conventional radiography $(\mathrm{CR})$ as they avoid the use of ionizing radiation.

The radiologist dealing with hepatobiliary and pancreatic diseases in children should be familiar with its various manifestations specific for this age group. There are diseases that are almost exclusively found in children, such as congenital anomalies and certain types of tumours. On the other hand, the child may present with an entity also found in adults with similar imaging appearances (e.g., cholelithiasis and hepatic cirrhosis), although the causative agent may be different and again age-specific. Knowledge of all the clinical and imaging characteristics is pivotal for the choice of the best imaging modality or combination of modalities, but also for the recognition of the diseases affecting children.

In this review we will discuss the technique of US, CT and MRI of the paediatric hepatobiliary and pancreatic system. Furthermore, we will address the imaging characteristics of several non-neoplastic hepatobiliary and pancreatic disease entities most common in children.

\section{Imaging techniques}

US

US is the imaging modality of choice for initial evaluation of children with suspected hepatobiliary or pancreatic diseases for many reasons. It is a patient-friendly and radiation-free imaging modality that does not suffer from motion artefacts. The relative small size of the child and fatfree intra-abdominal content results in improved penetration of the sound waves compared to adults, increasing the image quality. Furthermore, the ubiquitous availability of high-frequency transducers allows for a better tissue 
Table 1 Normal liver length in children [1]

\begin{tabular}{llcl}
\hline & \multicolumn{2}{l}{ Liver length in midclavicular line $(\mathrm{cm})$} \\
\cline { 2 - 4 } $\begin{array}{l}\text { Age } \\
\text { years })\end{array}$ & $\begin{array}{l}\text { Number of } \\
\text { patients }\end{array}$ & $\begin{array}{l}\text { Mean (standard } \\
\text { deviation[sd]) }\end{array}$ & $\begin{array}{l}\text { Limits of } \\
\text { normal }\end{array}$ \\
\hline $0-0.25$ & 53 & $6.4(1.0)$ & $4.0-9.0$ \\
$0.25-0.5$ & 40 & $7.3(1.1)$ & $4.5-9.5$ \\
$0.5-0.75$ & 20 & $7.9(0.8)$ & $6.0-10.0$ \\
$1-2.5$ & 18 & $8.5(1.0)$ & $6.5-10.5$ \\
$3-5$ & 27 & $8.6(1.2)$ & $6.5-11.5$ \\
$5-7$ & 30 & $10.0(1.4)$ & $7.0-12.5$ \\
$7-9$ & 38 & $10.5(1.1)$ & $7.5-13.0$ \\
$9-11$ & 30 & $10.5(1.2)$ & $7.5-13.5$ \\
$11-13$ & 16 & $11.5(1.4)$ & $8.5-14.0$ \\
$13-15$ & 23 & $11.8(1.5)$ & $8.5-14.0$ \\
$15-17$ & 12 & $12.1(1.2)$ & $9.5-14.5$ \\
\hline
\end{tabular}

differentiation and real-time evaluation of vascularisation (Doppler studies) and movements (e.g., peristalsis) is possible. Finally, the direct contact with the patient during the examination offers a unique opportunity to ask specific questions and perform additional physical examinations, emphasizing the role of the radiologist as a clinician.

Initially patients are scanned in the supine position after a (roughly $3 \mathrm{~h}$ ) period of fasting. To visualize the entire liver and pancreas additional positions (e.g., lateral decubitus) and additional manoeuvres (forced inspiration, graded compression, introduction of water into the stomach) may be necessary.

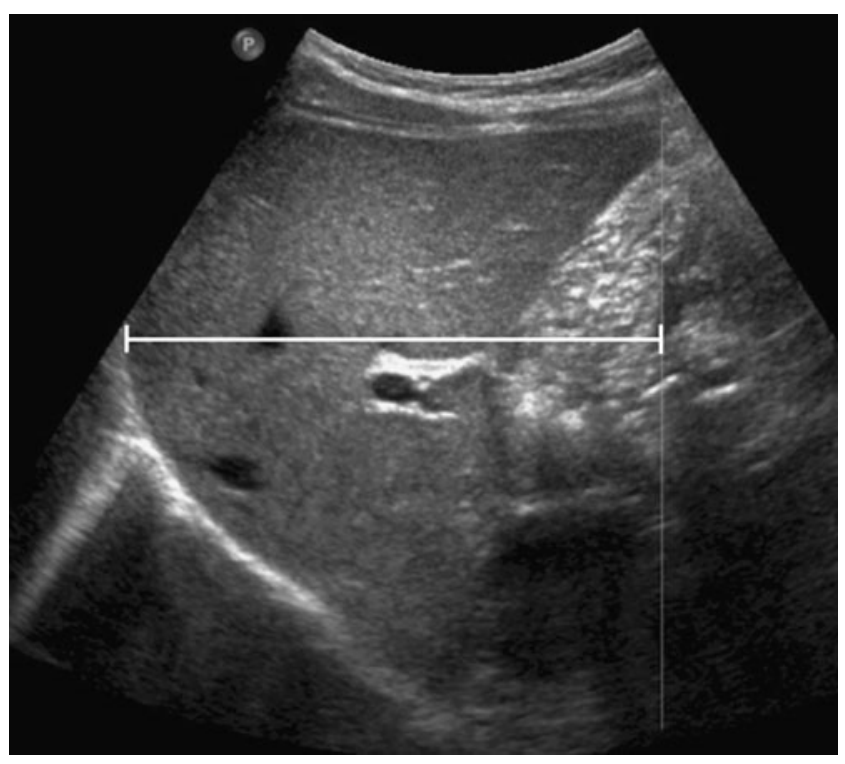

Fig. 1 Sagittal US image in the midclavicular line shows how to measure the length of the liver in children
Table 2 Normal values of the pancreas in children [2]

Maximal AP diameter pancreas (sd) in $\mathrm{cm}$

\begin{tabular}{llll}
\cline { 2 - 4 } Age & Head & Body & Tail \\
\hline Newborn infants & $1.0(0.4)$ & $0.6(0.2)$ & $1.0(0.4)$ \\
1 month-1 year & $1.5(0.5)$ & $0.8(0.3)$ & $1.2(0.4)$ \\
$1-5$ years & $1.7(0.3)$ & $1.0(0.2)$ & $1.8(0.4)$ \\
$5-10$ years & $1.6(0.4)$ & $1.0(0.3)$ & $1.8(0.4)$ \\
$10-19$ years & $2.0(0.5)$ & $1.1(0.3)$ & $2.0(0.4)$ \\
\hline
\end{tabular}

\section{Hepatobiliary imaging}

The liver is the largest solid organ in the upper abdomen. It has a homogeneous texture on US and is usually slightly hyperechoic to the kidney and slightly hypoechoic to the spleen. The following parameters have to be evaluated: echogenicity of the liver compared to renal cortex, size, shape and contours, texture, inferior caval vein, hepatic artery, portal vein, periportal echogenicity, gallbladder (size, contents and wall thickness), common bile duct and intrahepatic bile ducts. Table 1 summarizes the normal values for the liver length in children and Fig. 1 illustrates how to measure the liver [1].

\section{Pancreatic imaging}

The pancreas is a multilobular gland extending from the second portion of the duodenum to the splenic hilum. The parenchyma has a homogeneous texture. Its echogenicity is iso- or hyperechoic to the liver in the majority of children. In only $10 \%$ of children is it hypoechoic to the liver [2].

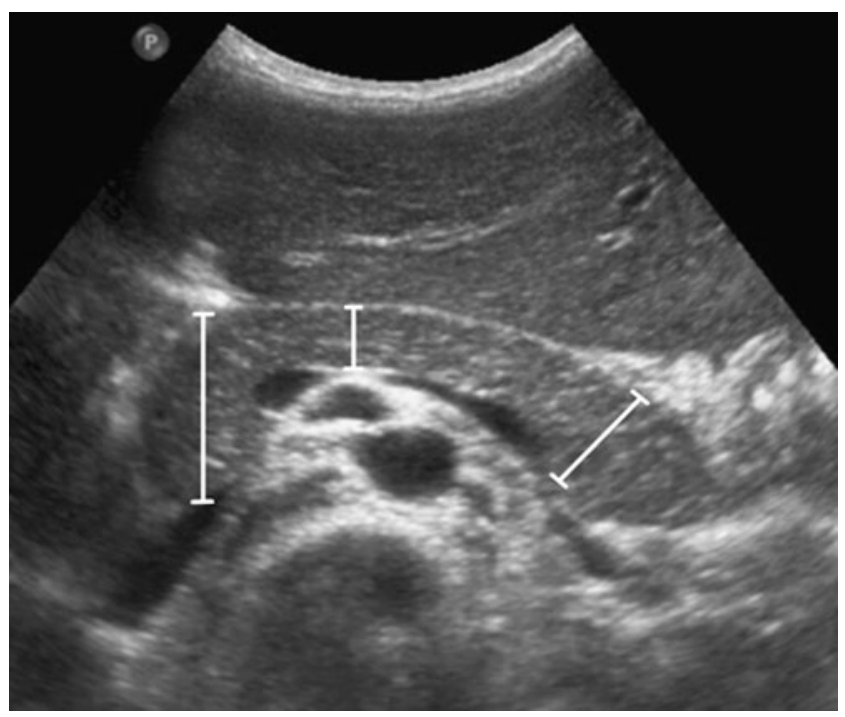

Fig. 2 Transverse US image shows the measurements of the head, body and tail of the pancreas in children 
Table 3 Guidelines for tube voltage $(\mathrm{kVp})$ and effective tube current $(\mathrm{mA})$ for 16 -slice CT scanners [6] *tube voltage $(\mathrm{kVp})$ for Philips scanners between brackets

\begin{tabular}{llllllc}
\hline Weight & CTDIvol & $\mathrm{kV}$ k $^{*}$ & $\begin{array}{l}\text { GE } \\
16 \times 1.25 \mathrm{~mm}\end{array}$ & $\begin{array}{l}\text { Philips } \\
16 \times 0.75 \mathrm{~mm}\end{array}$ & $\begin{array}{l}\text { Siemens } \\
16 \times 0.75 \mathrm{~mm}\end{array}$ & $\begin{array}{l}\text { Toshiba } \\
16 \times 1 \mathrm{~mm}, \mathrm{M}\end{array}$ \\
\hline $4-9 \mathrm{~kg}$ & 2.1 & $100(90)$ & 80 & 120 & 80 & 50 \\
$10-19 \mathrm{~kg}$ & 3.0 & $100(90)$ & 110 & 170 & 115 & 70 \\
$20-29 \mathrm{~kg}$ & 3.8 & $100(90)$ & 140 & 215 & 145 & 90 \\
$30-39 \mathrm{~kg}$ & 4.1 & $100(90)$ & 150 & 235 & 155 & 95 \\
$40-49 \mathrm{~kg}$ & 4.9 & 120 & 100 & 125 & 120 & 95 \\
$50-64 \mathrm{~kg}$ & 5.9 & 120 & 120 & 150 & 140 & 115 \\
\hline
\end{tabular}

Systematic scanning of the pancreas should include evaluation of size, texture, echogenicity, presence of calcifications or cysts, diameter of the pancreatic duct and the presence of surrounding fluid or oedema. In Table 2 the normal values for the pancreas are given and Fig. 2 illustrates how to make these measurements [2].

Optimal use of sonographic equipment is mandatory; to optimize imaging the frequency of the emitted sound waves should be as high as possible. Further optimization includes graded compression, optimal focus adjustments, compound imaging, adaptive image processing, harmonic imaging and in selected cases elastography or intravenous ultrasonographic contrast. Transient elastography is a relatively novel noninvasive method to detect and quantify liver fibrosis and/or cirrhosis [3]. This technique is performed using a US transducer in combination with a vibrator. By using the pulse-echo US acquisition it becomes possible to follow the propagation of the vibrations transmitted toward the liver tissue from the vibrator and measure their velocities that are related directly to the tissue stiffness. Recent studies suggest that this technique provides an objective and reproducible measure of the liver stiffness with a good interobserver agreement [3-5].

\section{CT}

The recent advances in multidetector CT (MDCT) have contributed to a substantial increase in its use and diagnostic accuracy, even in children. A major drawback of this development in MDCT, however, is the increasing use of ionizing radiation and, consequently, the risks of radiation-induced side effects of which the induction of secondary cancer is the most important. Therefore, MDCT should only be used when properly indicated (justification). Furthermore, if CT is indicated, the technique should be optimized in order to keep the radiation dose as low as reasonably achievable (ALARA).

Common indications for which MDCT is still used in children are trauma and staging of malignancies. Although for the latter MRI is increasingly validated and used (see below), CT still plays a role, especially when combined with FDG-PET. All other indications can usually be addressed by US and MRI. This is especially true for the characterization of liver lesions and the evaluation of the pancreaticobiliary system.

Optimization of paediatric MDCT starts with adequate prescan patient preparation [6]. This includes adequately

Table 4 Guidelines for MR imaging of the liver and pancreas (1.5T, Achieva, Philips Healthcare, Best, The Netherlands). NSA, number of acquisitions

\begin{tabular}{|c|c|c|c|c|c|c|c|}
\hline Pulse sequence & Plane & $\begin{array}{l}\mathrm{TE} \\
(\mathrm{ms})\end{array}$ & $\begin{array}{l}\text { TR } \\
(\mathrm{ms})\end{array}$ & $\begin{array}{l}\text { Flip angle } \\
\text { (degree) }\end{array}$ & NSA & $\begin{array}{l}\text { Slice thickness } \\
(\mathrm{mm})\end{array}$ & Comments \\
\hline $\begin{array}{l}\text { Breath-hold spoiled } \\
\text { gradient-echo (GRE) T1 }\end{array}$ & $\begin{array}{l}\text { Axial, sagittal } \\
\text { and/or } \\
\text { coronal }\end{array}$ & 4.2 & 8.5 & 10 & 1 & $\begin{array}{l}10 \mathrm{~mm} \text { (liver) } \\
10 \mathrm{~mm} \text { (pancreas) }\end{array}$ & \\
\hline $\begin{array}{l}\text { Breath-hold spoiled } \\
\text { GRE T1 in-phase }\end{array}$ & Axial & 4.6 & 181 & 80 & 2 & $\begin{array}{l}7 \mathrm{~mm} \text { (liver) } \\
7 \mathrm{~mm} \text { (pancreas) }\end{array}$ & TE $6.9 \mathrm{~ms}(1.0 \mathrm{~T})$ \\
\hline $\begin{array}{l}\text { Breath-hold spoiled } \\
\text { GRE T1 out-phase }\end{array}$ & Axial & 2.3 & 181 & 80 & 2 & $\begin{array}{l}7 \mathrm{~mm} \text { (liver) } \\
7 \mathrm{~mm} \text { (pancreas) }\end{array}$ & $\begin{array}{l}\text { TE } 3.45 \mathrm{~ms}(1.0 \\
\text { T) }\end{array}$ \\
\hline $\begin{array}{l}\text { Respiratory-triggered } \\
\text { (rt) turbo spin-echo } \\
\text { (TSE) T2 }\end{array}$ & Axial & 80 & 556 & 90 & 1 & $\begin{array}{l}7 \mathrm{~mm} \text { (liver) } \\
7 \mathrm{~mm} \text { (pancreas) }\end{array}$ & \\
\hline Rt spoiled GRE T1 & Axial & 4.6 & 10 & 15 & 1 & $\begin{array}{l}7 \mathrm{~mm} \text { (liver) } \\
7 \mathrm{~mm} \text { (pancreas) }\end{array}$ & \\
\hline $\begin{array}{l}\text { Breath-hold 3-D } \\
\text { spoiled GRE T1 with } \\
\text { fat saturation }\end{array}$ & $\begin{array}{l}\text { Axial } \\
\text { Axial }\end{array}$ & $\begin{array}{l}1.9 \\
2.1\end{array}$ & $\begin{array}{l}3.9 \\
4.3\end{array}$ & $\begin{array}{l}10 \\
10\end{array}$ & $\begin{array}{l}1 \\
1\end{array}$ & $\begin{array}{l}4 \mathrm{~mm} \text { (liver) } \\
4 \mathrm{~mm} \text { (pancreas) }\end{array}$ & $\begin{array}{l}\text { dynamic } \\
\text { multiphase } \\
\text { sequence after } \\
\text { IV contrast agent }\end{array}$ \\
\hline
\end{tabular}


Table 5 Guidelines for MRCP (1.5T, Achieva, Philips Healthcare, Best, The Netherlands)

\begin{tabular}{|c|c|c|c|c|c|c|c|}
\hline Pulse sequence & Plane & TE (ms) & TR (ms) & $\begin{array}{l}\text { Flip angle } \\
\text { (degree) }\end{array}$ & NSA & $\begin{array}{l}\text { Slice thickness } \\
(\mathrm{mm})\end{array}$ & Comments \\
\hline $\begin{array}{l}\text { Breath-hold } \\
\text { spoiled GRE T1 }\end{array}$ & $\begin{array}{l}\text { Axial, sagittal } \\
\text { and/or coronal }\end{array}$ & 4.2 & 8.5 & 10 & 1 & $10 \mathrm{~mm}$ & \\
\hline Rt TSE T2 & Axial & 80 & 448 & 90 & 2 & $7 \mathrm{~mm}$ & \\
\hline Rt spoiled GRE T1 & Axial & 4.6 & 10 & 15 & 2 & $7 \mathrm{~mm}$ & \\
\hline $\begin{array}{l}\text { Breath-hold 3-D } \\
\text { TSE T2 (thick slab) }\end{array}$ & Coronal & 800 & 8000 & 90 & 1 & $40 \mathrm{~mm}$ & $\begin{array}{l}\text { nine slices with a } \\
\text { radial acquisition } \\
\text { and pancreatic } \\
\text { head in centre }\end{array}$ \\
\hline Rt 3-D TSE T2 & Coronal & 650 & 1204 & 90 & 1 & $0.8 \mathrm{~mm}$ & MIP reconstructions \\
\hline
\end{tabular}

NSA, number of acquisitions

informing the child and parents about the procedure, adaptation of the scanner environment, addressing the need for sedation and/or anaesthesia, and several (intravenous) contrast issues. Subsequently, the various scan and technical parameters should be tailored to the size of the child, body region of interest and the clinical question. Multiphase CT examinations should be avoided. An empirically determined fixed-delay time of $50 \mathrm{~s}$ after initiation of the IV injection of contrast material usually suffices, resulting in a $\mathrm{CT}$ examination during the portal venous phase. In most children a tube voltage of $80-100 \mathrm{kVp}$ can be chosen and only in adolescents is $120 \mathrm{kVp}$ required in the abdomen. The tube current $(\mathrm{mA})$ should be adapted to the size or weight of the child, as illustrated in Table 3. Although an increase in pitch can result in a shorter scan time, it appears to be more dose efficient to keep the pitch as low as possible (usually $<1$ ), with as extra benefit a better spatial resolution. In addition, the scan field of view should be tailored to the size of the upper abdomen with as major advantage a higher spatial resolution. Most modern MDCT scanners have tube rotation times between 0.3 and $0.5 \mathrm{~s}$ resulting in shorter scan times. In terms of image quality a rotation time of $0.5 \mathrm{~s}$ is often the best option. The tube current modulation techniques, available on almost all modern MDCT scanners, are increasingly used in paediatric MDCT, although specific paediatric settings have limited availability. However, one should realise that the (extra) amount of radiation dose reduction is often limited or absent when the CT protocols already have been optimized by using fixed tube current $(\mathrm{mA})$ tables adapted to the size or weight of the child.

\section{MRI}

Common indications for MRI are (the characterization of) liver and pancreatic tumours, inflammatory or infectious conditions and vascular malformations. As stated before, MRI is increasingly used for oncological indications, not only because of its excellent tissue contrast and resolution but also because of the promising advances in functional imaging techniques such as diffusion-weighted imaging (DWI) and MR-spectroscopy (MRS) [7]. MR cholangiopancreatography (MRCP) has also proved to be very useful
Fig. 3 Persistent neonatal cholestasis and biopsy-proven biliary atresia in a 1.5-month-old boy. US shows the triangular cord sign (arrow in a) and increased diameter of the hepatic artery $(27 \mathrm{~mm}$, arrow in $\mathbf{b}$ )
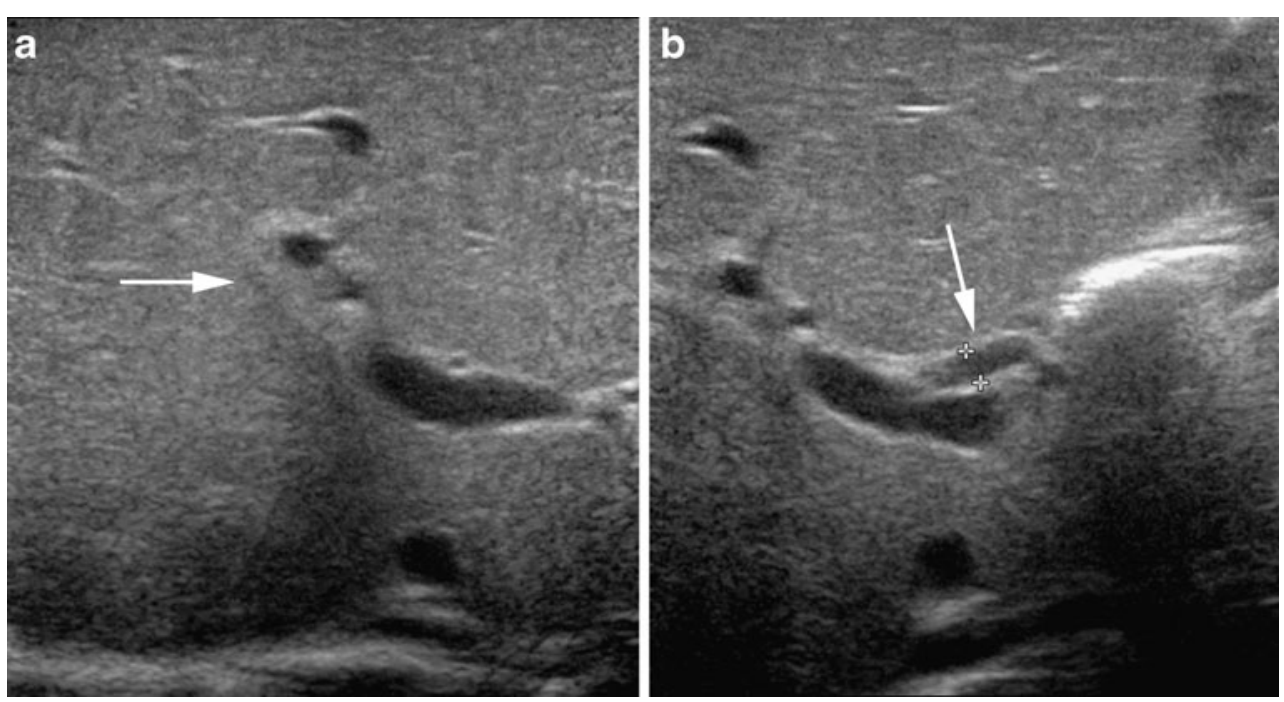


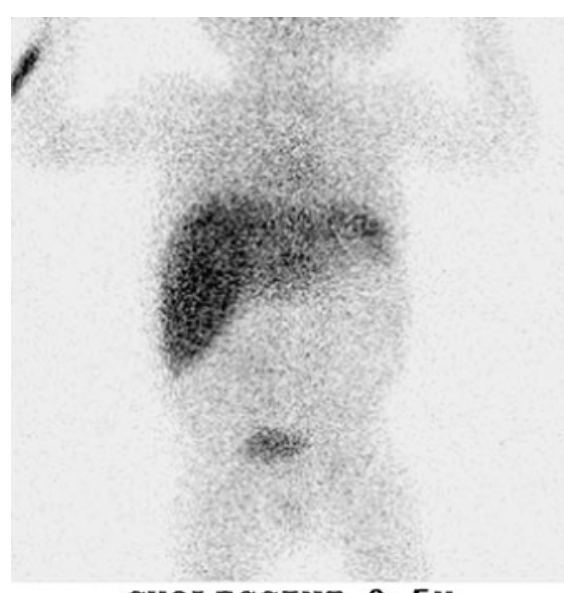

CHOLESCINT $2.5 \mathrm{H}$

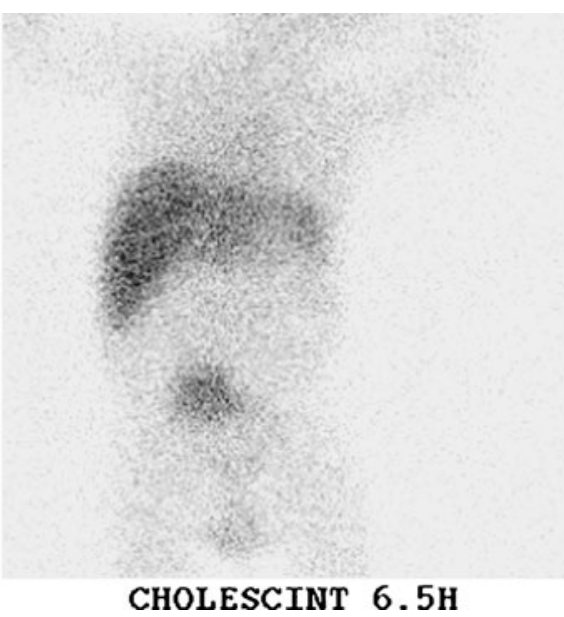

CHOLESCINT $6.5 \mathrm{H}$

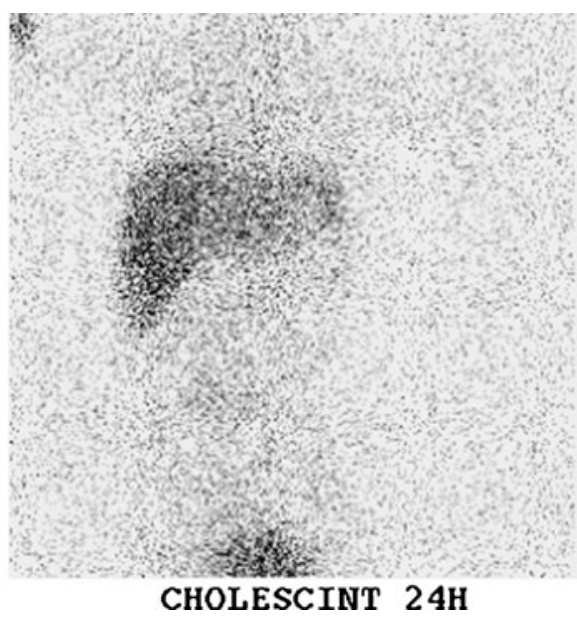

CHOLESCINT 24H

Fig. 4 IDA scintigraphy in the same patient as Fig. 3. There is a normal hepatic extraction of the tracer, but no excretion into the GI tract, even on delayed imaging at $24 \mathrm{~h}$ after injection of the tracer

in children. Indications are choledochal duct malformations and posttraumatic, postoperative or postinflammatory changes of the pancreaticobiliary tree.

Although most MRI investigations in children have to be planned individually and tailored to the clinical question as well as the age, size and cooperation of the child, some general guidelines for an imaging protocol can be given [79]. MRI of the liver or pancreas is usually performed using a head or body phased-array coil depending on the size of the child. The standard protocol at least consists of T1- and T2-weighted (turbo) spin-echo (TSE) sequences in the axial plane, usually with breath-hold or respiratory gating. Other planes can be helpful but it is seldom necessary to image in all three orthogonal planes. Fat-saturation can be applied and is especially useful in the post-gadolinium imaging. Dynamic gadolinium-enhanced 3-D fast spoiled gradientecho (GRE) sequences are obtained in case of MR angiography or venography, and tumour imaging. In- and out-of-phase sequences can be used to assess the fatty content of a lesion. DWI is only used in selected cases, such as tumour imaging. Table 4 gives an overview of the different sequences available with guidelines for some technical parameters.

When performing a MRCP, fasting for $3-5 \mathrm{~h}$ prior to the examination is essential in order to fully distend the gallbladder and biliary system, empty the stomach of
Fig. 5 Images in a 19-year-old woman with a history of biliary atresia and Kasai procedure (hepatic portoenterostomy), now presenting with sepsis and cholangitis. a Coronal MPR of the CT scan of the abdomen shows massive splenomegaly, irregular dilatation of the biliary tree (arrowheads) and a complex multiloculated fluid collection (biloma) with the suggestion of a connection with the biliary tree (arrow). b MIP reconstruction of the rt 3-D TSE T2-W sequence also shows the portoenterostomy (arrowheads), irregular dilatation of the biliary tree (closed arrow) and the close relation of the biliary tree to the biloma (open arrow). Furthermore, there is ascites visible surrounding the liver
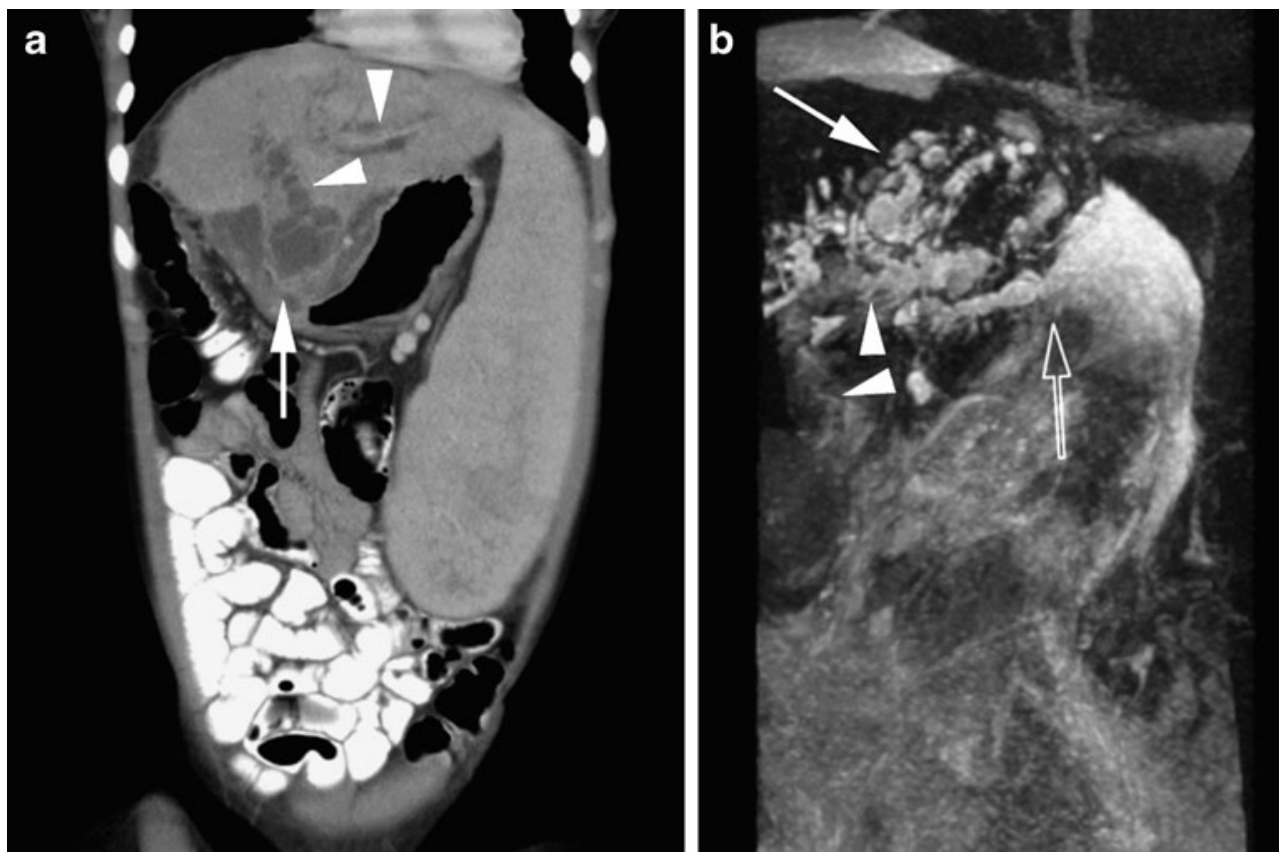

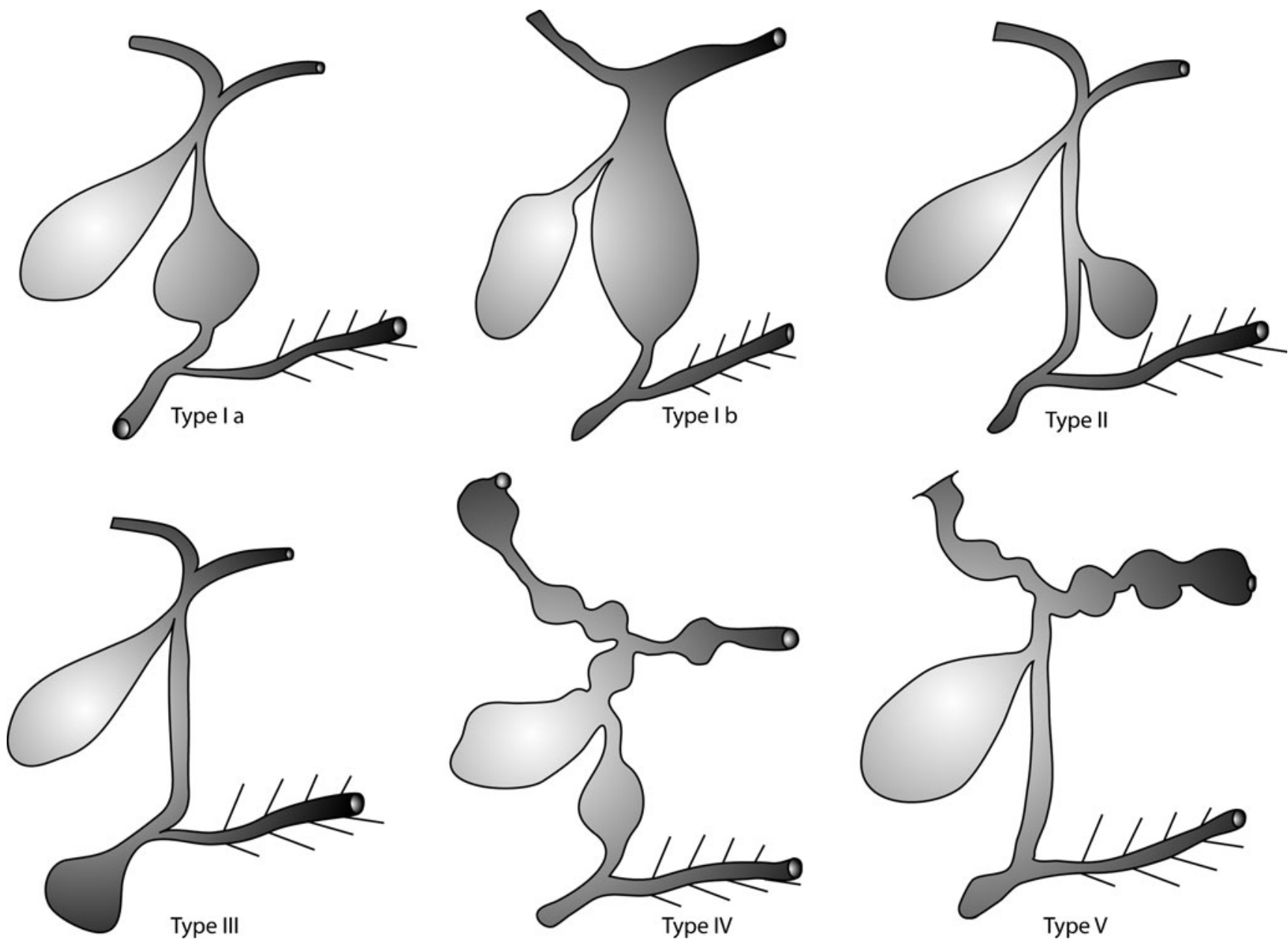

Fig. 6 Schematic representation of the classification of choledochal cysts according to Todani: type I, fusiform dilatation of the common bile duct below the cystic bile duct (Ia), or of the common bile duct and main hepatic ducts (Ib); type II, one or more cystic diverticula of the common bile duct; type III, focal dilatation of the distal common

bile duct in the papillary region into which the pancreatic ducts drain (also called choledochoceles); type IV, multiple dilatations of the intraand extrahepatic (IVa) or only the extrahepatic (IVb) bile ducts; and type V, Caroli disease (segmental ectasia of the large intrahepatic ducts throughout the liver)

contents and reduce intestinal motility $[10,11]$. The use of an oral negative paramagnetic agent (e.g., ferumoxsil and ferric ammonium citrate) to suppress fluid in the proximal duodenum and small intestine is possible [11, 12]. Non- breath-hold T2-W fast spin-echo 2- or 3-D sequences with respiratory gating are especially useful in infants and young children. A regular breathing pattern is required. The acquisition plane is coronal. If maximum-intensity projec-
Fig. 7 Images in a 2-month-old girl presenting with an antenatally diagnosed (asymptomatic) cyst in the region of the liver. US shows (a) cystic dilatation of the common bile duct, extending from the pancreatic head up to the level where the cystic duct enters the common bile duct. b A type I choledochal cyst
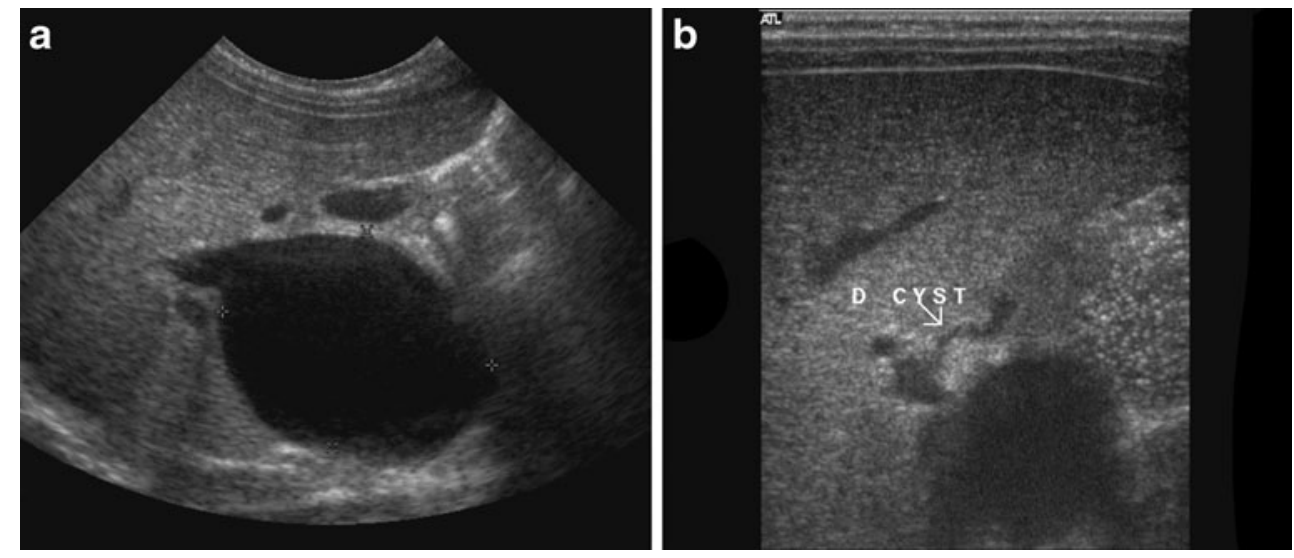
Fig. 8 Antenatally diagnosed liver cysts and enlarged kidneys in a 4-day-old boy. a US of the liver shows multiple fusiform and cystic dilatations of the intrahepatic bile ducts. One of the cysts shows the "central dot" sign, representing the portal fibrovascular bundle (arrow); type V choledochal cysts. b US of the kidneys shows enlargement and increased echogenicity with multiple small cysts characteristic of polycystic kidney disease; Caroli disease
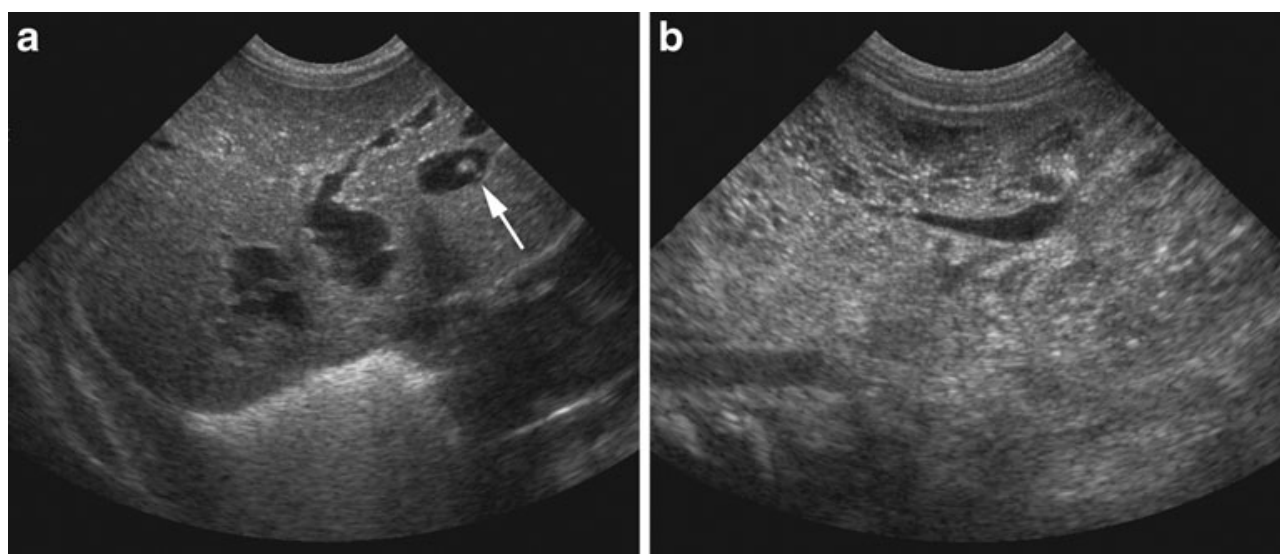

tions (MIP) will be reconstructed longer echo time values and thin $(<1 \mathrm{~mm})$ overlapping slices are preferred. Breathhold techniques, including single thick-slab and multiple thin-slab sequences, are faster and more suitable for the older children. Heavily T2-W TSE sequences are used to acquire thick slabs up to $4 \mathrm{~cm}$ in the coronal plane. These thick slabs can be repeated at multiple degrees of obliquity. Multiple thin (3-4 mm) slices can be obtained with half-Fourier single-shot (HASTE) or single-shot fast spin-echo (SSFSE) sequences. The HASTE technique is the preferred method as it is superior to the heavily

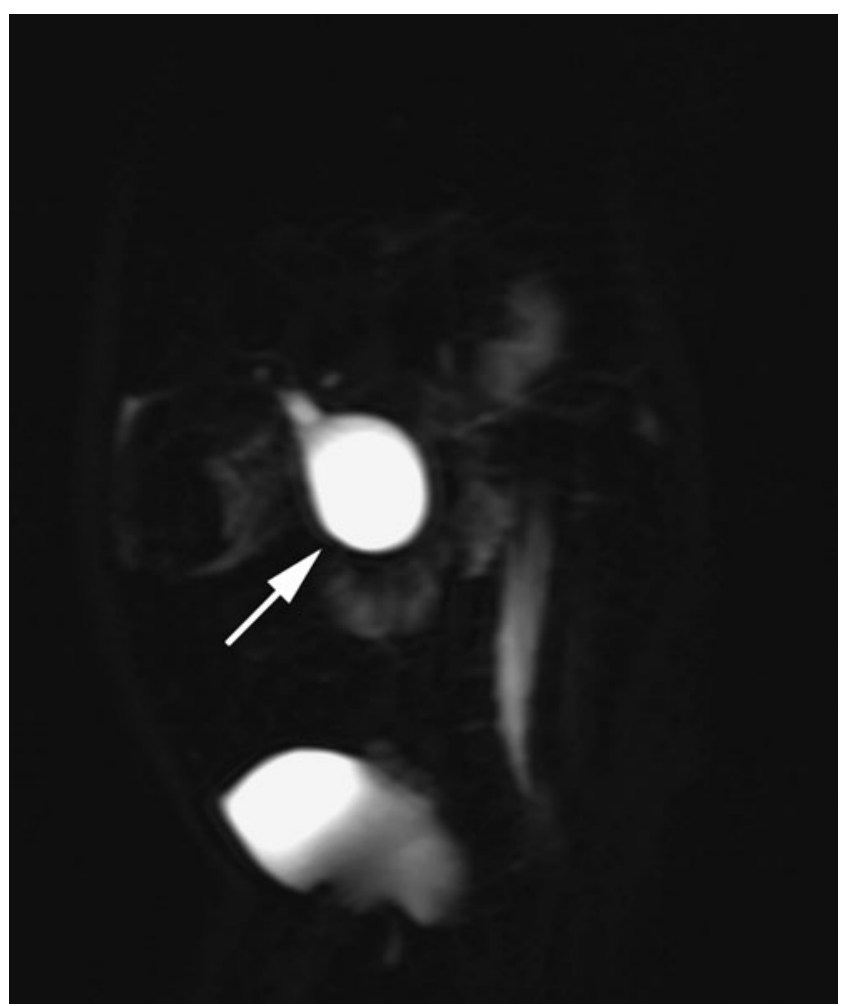

Fig. 9 MRCP in the same patient as Fig. 7. Oblique coronal thick slab image of a breath-hold 3-D TSE T2-W sequence clearly shows the type I choledochal cyst (arrow)
T2-W fast spin-echo techniques in demonstrating the pancreaticobiliary anatomy. In Table 5 the different sequences used for MRCP are summarized with guidelines for some technical parameters.

\section{Congenital anomalies}

\section{Biliary atresia}

Biliary atresia is a congenital obstruction of the intra- and/ or extrahepatic bile ducts [13-15]. It is one of the causes of persistent neonatal jaundice and is two times more frequent in males. The overall incidence is approximately 1:15,000 births, but it is far more common in the Asian population. Children with biliary atresia may have similar clinical, biochemical and histological manifestations as neonatal hepatitis and, therefore, diagnostic imaging plays an important role in differentiating these and other causes of jaundice.

US is the imaging modality of choice to initially evaluate jaundiced neonates. In biliary atresia, one of the most common findings is an absent, small $(<1.5 \mathrm{~cm})$ or empty gallbladder, assuming that the neonate has been fasting for several hours. Absence of the gallbladder occurs in approximately two-thirds of neonates with biliary atresia [16]. Another important sonographic finding is the triangular cord sign, which is defined as a triangular or tubeshaped echogenic focus at the porta hepatis that follows the portal veins and measures more than $4 \mathrm{~mm}$ in thickness (Fig. 3) [16-18]. According to the recent literature, this sign has a sensitivity of $62-93 \%$ and a specificity of $96-100 \%$. However, it may be difficult to distinguish this sign from diffuse periportal echogenicity due to inflammation or cirrhosis. Other signs that may be helpful in the diagnosis of biliary atresia are an absent common bile duct (reported sensitivity $93 \%$ and specificity $95 \%$ ) and a hypertrophic hepatic artery (reported diameter $2.2 \pm 0.59 \mathrm{~mm}$ ) (Fig. 3). Finally, Lee et al. [18] recently published the finding of 
Fig. 10 US in a 10-year-old girl with CF. a Transverse US image shows inhomogeneous echogenicity of the liver parenchyma due to liver fibrosis and steatosis. b This detailed transverse US image with a high frequency $(12-5 \mathrm{mHz})$ linear transducer nicely illustrates the undulating contours of the liver parenchyma (arrow) suggesting progression of the liver fibrosis to cirrhosis
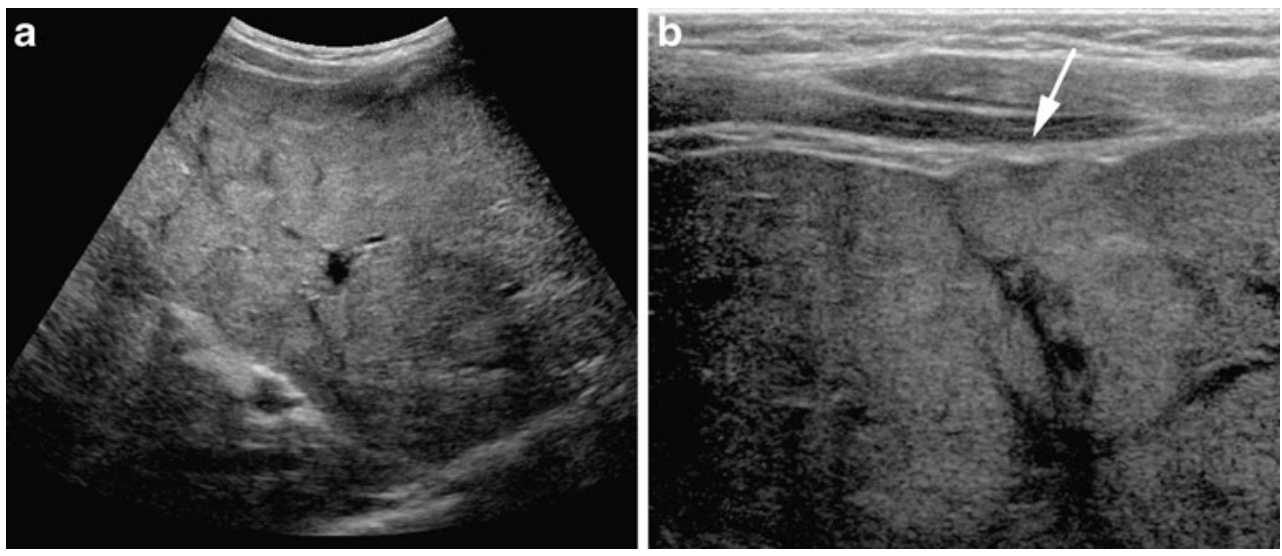

increased hepatic subcapsular flow on color Doppler US with a sensitivity of $100 \%$ and specificity of $86 \%$.

Hepatobiliary scintigraphy (HBS) with ${ }^{99 \mathrm{~m}}$ Tc-labeled iminodiacetic acid (IDA) analogues may play a role in the evaluation of biliary atresia, although its role has decreased with the improvements in US and image-guided biopsies. Premedication with oral phenobarbital for at least $5 \mathrm{~d}$ prior to the imaging improves the diagnostic accuracy. The combined technique of HBS with SPECT and phenobarbital has the highest accuracy (up to 98\%) [19, 20]. It usually permits accurate differentiation of biliary atresia from other causes of neonatal jaundice. In biliary atresia there is usually a normal or rapid hepatic extraction of the tracer, but no excretion into the gastrointestinal (GI) tract, even on delayed imaging up to $24 \mathrm{~h}$ (Fig. 4). In a small percentage of patients, however, there may be a conjoined poor hepatic function resulting in poor hepatic extraction and poor or no excretion in the GI tract, and these imaging findings overlap with hepatitis. In other words, although the sensitivity of scintigraphy is high (up to $100 \%$ ), its specificity is reported to vary between $43 \%$ and $87.5 \%$ [21]. This is mainly due to the fact that children with neonatal hepatitis also may show a non-excreting IDA scintigram. HBS is therefore most useful in determining patency of the biliary tract, thereby excluding biliary atresia.

MRCP may be used for the evaluation of neonatal jaundice. Obtaining diagnostic images in neonates will be challenging, however, because of the motion sensitivity related to the rapid respiratory and heart rate, as well as the poor visibility of the biliary ducts due to the small size, fibrosis, and little intraluminal fluid. Therefore, it is best utilized as a problem-solving technique and only when the biliary tree is dilated (Fig. 5).

\section{Choledochal cysts}

Choledochal cysts are congenital saccular or fusiform dilatations of the biliary tree and are approximately three times more prevalent in females [15]. The overall incidence is approximately 1-2:100,000-150,000 live births, but again they are far more common in the Asian population with a reported prevalence of up to 1:1,000 in Japan [22, 23]. Choledochal cysts are usually classified according to Todani [24] into five types (Fig. 6): type I, fusiform dilatation of the common bile duct below the cystic bile duct (Ia), or of the common bile duct and hepatic ducts (Ib); type II, one or more cystic diverticula of the common bile ducts; type III, focal dilatation of the distal common bile duct in the papil region into which the pancreatic ducts drain (also called choledochoceles); type IV, multiple dilatations of the intraand extrahepatic (IVa) or only the extrahepatic (IVb) bile ducts; and type V, Caroli disease (segmental ectasia of the large intrahepatic ducts throughout the liver). The type I choledochal cysts are the most common, occurring in up to $80-90 \%$ of cases. An abnormal junction of the common bile duct with the pancreatic duct is associated with choledochal cysts, and found in up to $90 \%$ of cases in the Asian population [23].

Choledochal cysts often present with cholestatic jaundice, although in older children the typical triad of abdominal pain, fever and obstructive jaundice may be present $[22,25]$. The most common complication of choledochal cysts is an ascending cholangitis and/or pancreatitis, often caused by reflux of pancreatic secretions in the bile ducts due to the abnormal junction of common bile and pancreatic duct. Liver cirrhosis, portal hypertension and spontaneous cyst rupture are other reported complications. Children with choledochal cysts do have an increased risk of developing cholangiocarcinoma, especially over the age of 10 years. The incidence reported varies between $9 \%$ and $28 \%$ [25].

The initial imaging modality of choice is US $[9,26]$. The localisation and degree of bile duct dilatation can be easily identified with this technique (Fig. 7). Occasionally, sludge or bile stones are identified due to bile stasis in the dilated bile ducts. In case of type 5 choledochal cysts (Caroli disease) the kidneys should be examined as well, because 
Fig. 11 US and MRI in a 6-month-old girl with BeckwithWiedemann syndrome. a US shows multiple sharply demarcated hypoechoic lesions throughout the liver parenchyma (arrows). b Axial rt TSE T1-W and (c) rt TSE T2-W images confirm multiple focal liver lesions, dark on $\mathrm{T} 1$ and bright on T2. d, e Serial dynamic axial 3-D T1-W images after intravenous contrast administration show early,

peripheral nodular or complete enhancement of the lesions and during the late phase, complete fill-in without signs of wash-out. In the clinical setting of Beckwith-Wiedemann the MR signs are compatible with multiple hepatoblastomas or haemangiomas (final histological diagnosis: haemangiomas)
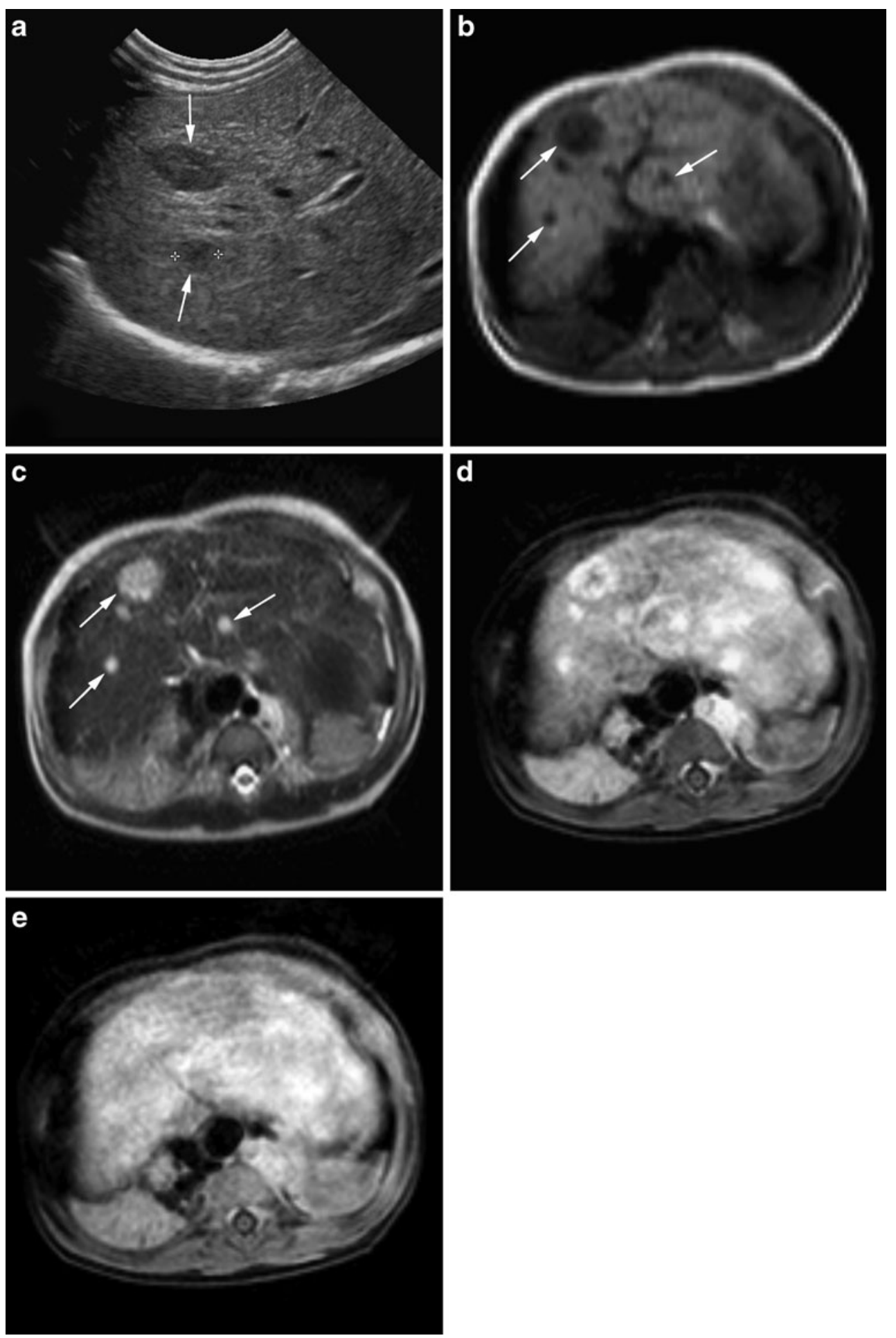

this type may be associated with autosomal-recessive polycystic kidney disease (Fig. 8). It may be difficult to differentiate choledochal cysts from a simple hepatic cyst, hepatic abscess or pancreatic pseudocyst. MRI and MRCP may be helpful in demonstrating the extent of the disease and relationship of the cysts to the surrounding tissues
(Fig. 9) [9-11, 26, 27]. The diagnostic accuracy of this technique varies between $82 \%$ and $100 \%$. MRCP may miss small biliary cysts in the periphery of the liver. Biliary scintigraphy with ${ }^{99 m}$ Tc-labeled IDA may play a role by demonstrating communication of the cyst(s) with the biliary tree due to the focal accumulation of activity in the cyst. 


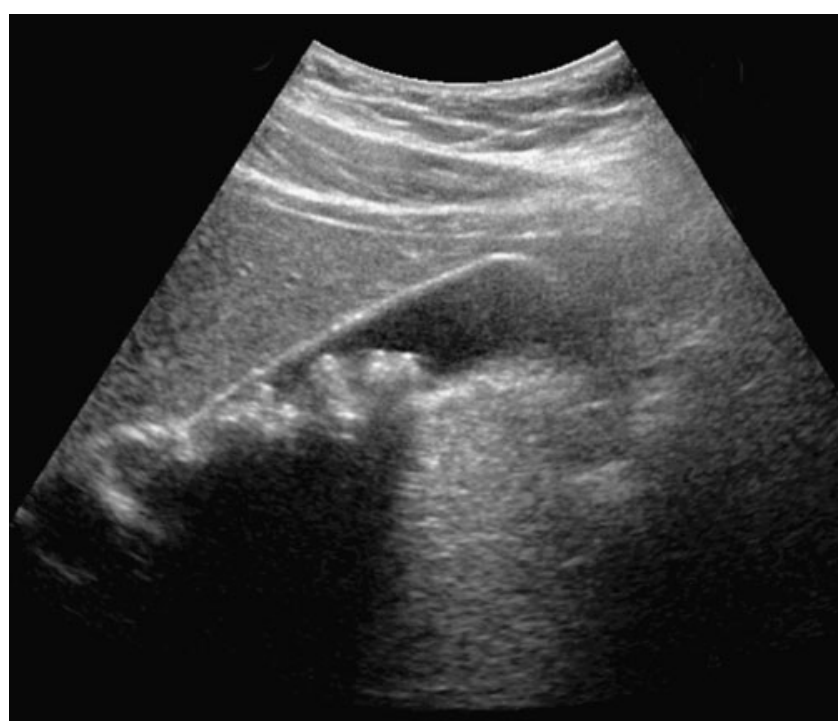

Fig. 12 US in a 16-year-old girl with colicky upper abdominal pain. There are multiple echogenic structures with acoustic shadowing visible within the gallbladder compatible with gallstones

\section{Developmental anomalies of the pancreas}

Congenital anomalies of the pancreas include pancreas divisum, annular pancreas, agenesis of the dorsal pancreatic anlagen, ectopic pancreatic tissue, hereditary chronic pancreatitis and nesidioblastosis [28]. Nesidioblastosis is persistence of the normal fetal state of the pancreas (diffuse proliferation and persistence of nesidioblasts). These cells secrete an excess of insulin causing hypoglycaemia. Neonatal cases of nesidioblastosis are characterized by diffusely increased echogenicity and increased size of pancreatic head, body and tail. Treatment is subtotal pancreatectomy.

Syndromes that can manifest with pancreatic pathology include: Beckwith-Wiedemann syndrome, Von HippelLindau disease and autosomal-dominant polycystic kidney disease. Children and adults with Shwachman-Diamond syndrome and cystic fibrosis (CF) frequently present with pancreatic insufficiency. In the next section on hereditary disorders a separate paragraph is dedicated to the pancreatic and liver manifestations of $\mathrm{CF}$.

\section{Hereditary disorders}

\section{$\mathrm{CF}$}

$\mathrm{CF}$ is an autosomal-recessive disease caused by a gene defect encoding for the CF transmembrane conductance regulator (CFTR) [29]. This abnormal chloride metabolism is manifested especially in the cells of exocrine glands, resulting in increased viscosity of the products of these cells. Affected are sweat glands, mucus producing cells in the tracheobronchial tree, intestinal tract, exocrine tissue of pancreas and the seminal vesicles in boys. In the liver CFTR is expressed only on the apical surface of the cells of the biliary epithelium, leading to dehydration of bile with obstruction of bile ducts. In patients with CF, liver and pancreas are gradually affected resulting in end-stage liver cirrhosis and pancreatic atrophy [30].

\section{Liver in $C F$}

Chronic liver disease is one of the major complications of CF [30]. Significant liver disease is seen in $13-25 \%$ of children with CF [31]. Liver disease typically develops in the first decade of life, with the incidence dropping rapidly after the age of 10 years. Histologically, three types of liver disease are seen in CF: steatosis, focal biliary fibrosis and multilobular cirrhosis.

US is the modality of choice in childhood [32]. At birth the liver is normal. The disease process is variable but there are some generalizations. The most common ultrasonographic finding is increased echogenicity of the liver in $50 \%$ of patients. This increased echogenicity is homogeneous in $70 \%$ and heterogeneous in 30\% (Fig. 10) [33]. Increased echogenicity is often caused by steatosis or focal
Fig. 13 US of the liver in a 2-month-old boy with giant cell hepatitis and cholestasis. US shows intrahepatic biliary dilatation (arrowheads in a), dilatation of the common bile duct (arrowheads in b) and an obstructive echogenic focus in the distal common bile duct at the level of the papilla of Vater (arrow in b), suspicious for an obstructive gallstone
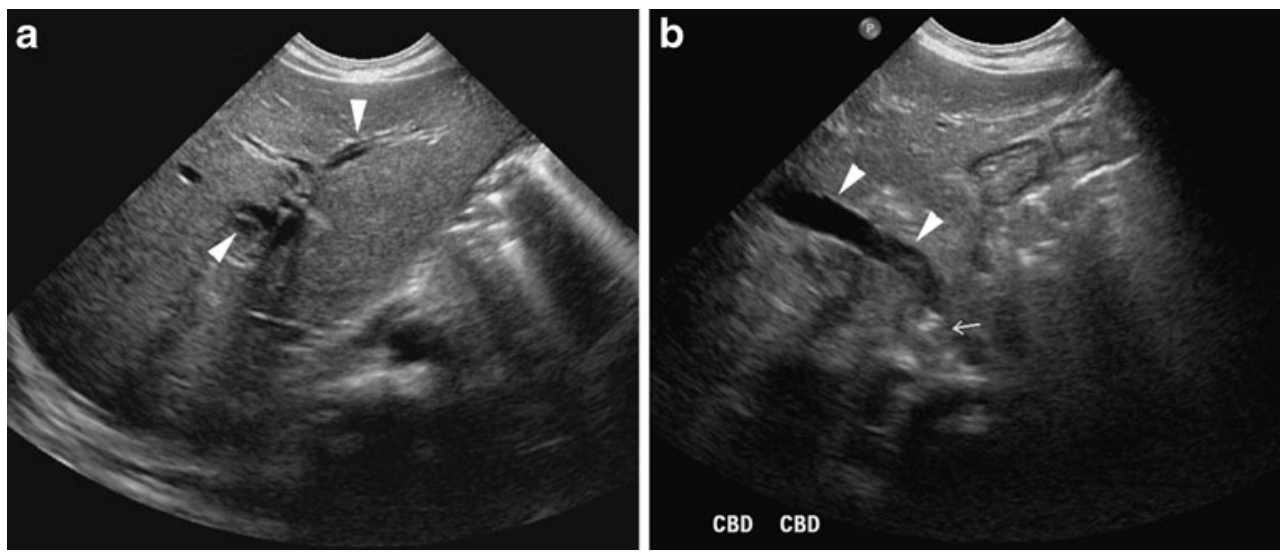


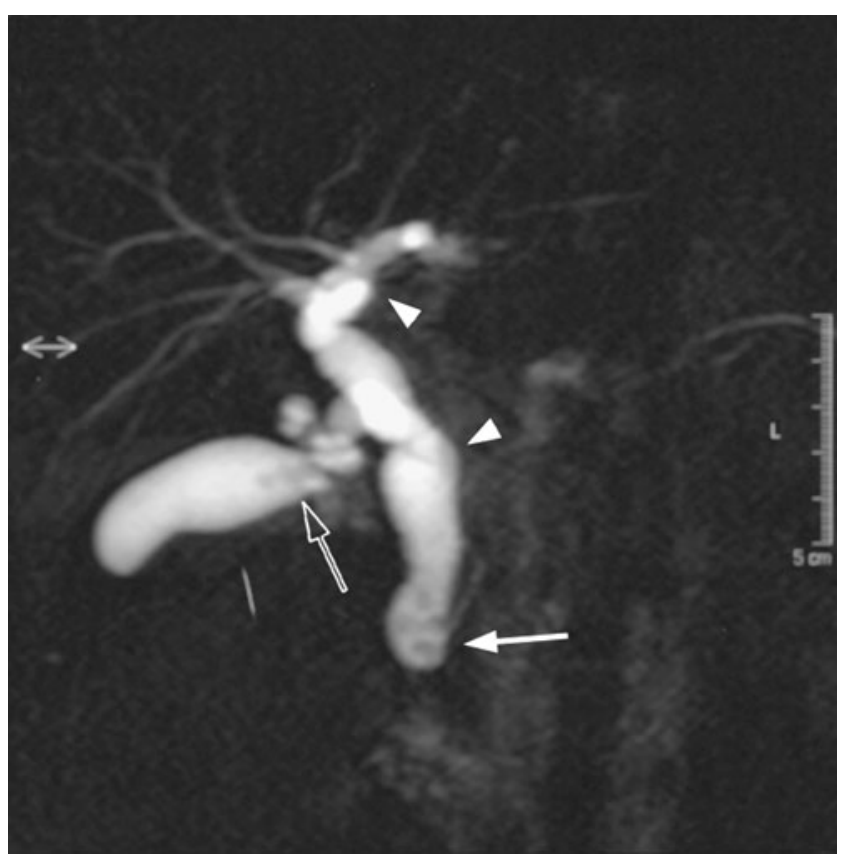

Fig. $14 \mathrm{MRCP}$ in a child with cholecysto- and choledocholithiasis. Oblique coronal thick slab image of a bh 3-D TSE T2-W sequence shows multiple filling defects in the gallbladder neck (open arrow) and distal in the common bile duct (closed arrow). Furthermore, there is dilatation of the common bile duct and main hepatic ducts (arrowheads)

biliary fibrosis. Prominent periportal echogenicity reflects morphological inflammatory changes caused by inspissated bile and is seen with ultrasonography in $37 \%$ of the children. The gallbladder atrophies or becomes filled with thickened bile or stones $(24 \%)$.

The first direct ultrasonographic sign of cirrhosis is nodularity of the liver. Before the nodules themselves become visible indirect signs of nodularity should be looked for, such as undulating contours of the liver surface or undulating contours of the endothelial lining of the liver veins (Fig. 10). Gradually the liver atrophies and portal hypertension develops. A study comparing US with biopsy findings showed that abnormal ultrasonographic findings predict fibrosis and cirrhosis, but normal ultrasonographic findings do not exclude it [34]. However, liver biopsy can be unreliable due to sampling errors caused by focal distribution of cirrhotic changes. Transient elastography may be an attractive alternative to noninvasively assess and follow-up CF-associated liver disease [5].

CT offers additional information but this does not justify its use as the initial imaging modality of choice. In patients with increased echogenicity CT may differentiate between steatosis and focal biliary fibrosis. Eventually malignant degeneration occurs but this is far beyond childhood. MRI can be considered as an alternative to US and CT, not in the least because it provides unique information of the biliary tree.

\section{Pancreas in $\mathrm{CF}$}

Pancreatic involvement in children with $\mathrm{CF}$ is more frequent than liver disease [35]. Plugging of pancreatic ducts with inspissated secretions is thought to play a major role in the pathogenesis. It results in exocrine insufficiency, with clinically apparent dysfunction in $85-90 \%$ of patients. Pancreatitis is a rare complication (1.2\% of patients). The morphological abnormalities in the pancreas are caused by fat deposition and fibrosis. US is the initial modality of choice, showing an enlarged or small pancreas with increased echogenicity. Areas of decreased echogenicity represent fibrosis.

CT and MRI can demonstrate these changes to a better advantage but are time-consuming, expensive or involve radiation (CT). Although MRI is superior in demonstrating fatty infiltration, it cannot demonstrate small calcifications that can occur in $\mathrm{CF}$.

\section{Beckwith-Wiedemann syndrome}

Beckwith-Wiedemann syndrome (also called EMG syndrome) is characterized by congenital abdominal wall defects (exomphalos), macroglossia and pre- and postnatal overgrowth (gigantism) [36, 37]. Additional findings may include organomegaly (usually liver and kidney), hypoglycaemia, hemihypertrophy and genitourinary abnormalities. Five- to twenty-percent of these children will develop embryonal tumours (most commonly Wilms tumor or hepatoblastoma), or adrenal tumors. Most of these tumours will develop in the first years of life, usually younger than 4 years of age, although Wilms tumor may occur until 8 years of age.

Because of the increased risk of developing a malignancy, longitudinal abdominal US is recommended, preferably at 3-month intervals during childhood. This is because of the rapid growth rate of some tumours, especially Wilms tumour. US will show organomegaly (usually hepatomegaly and nephromegaly), which may be confined to one side of the child (hemihypertrophy). Furthermore, tumours in one of the upper abdominal organs may be detected, especially in the kidney and liver (Fig. 11). CT can play a role in the work-up of detected tumours although MRI is increasingly validated and used for this.

\section{Acquired biliary pathology}

\section{Cholelithiasis}

Although gallstones are relatively uncommon in children, their incidence has been increasing over the last decades. This is mainly due to the more widespread use of US with, as a 

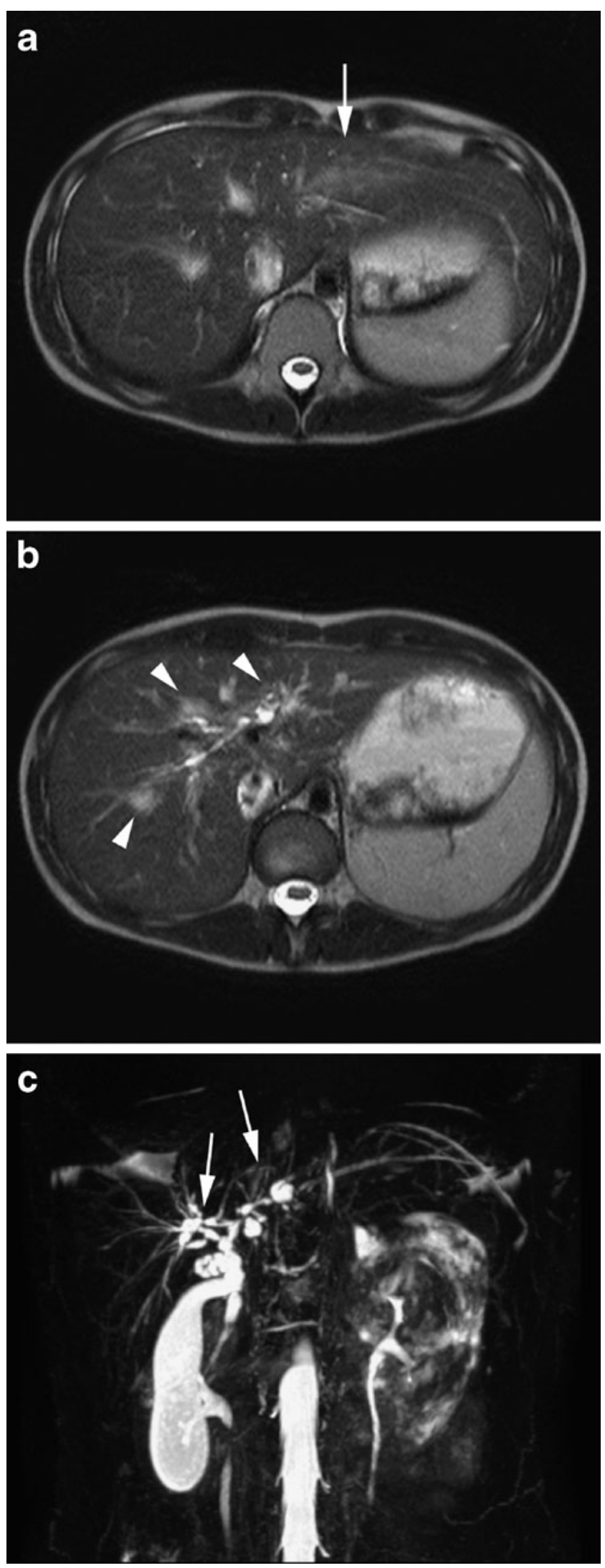

Fig. 15 MRI and MRCP in a 10-year-old girl with ulcerative colitis and signs of cholestasis. a, b Axial rt TSE T2-W images show a wedge-shaped area of T2 hyperintensity (arrow in a) as well as periportal parenchymal hyperintensities and biliary duct dilatations (arrowheads in b). c MIP reconstruction of the rt 3-D TSE T2-W sequence clearly illustrates the dilatations and strictures in the common bile duct and central intrahepatic bile ducts (arrows) compatible with primary sclerosing cholangitis

consequence, gallstones more frequently detected in asymptomatic children. Furthermore, the increase in obesity in the paediatric age-group certainly plays a role. In a recent population-based study the reported prevalence of gallstones in children was $1.9 \%$ [38]. Cholelithiasis may by idiopathic, but in the neonate and young infant it is often associated with sepsis, diuretics and total parenteral nutrition [39]. In older children, gallstone formation may be caused by haemolytic anaemia (e.g., sickle cell disease), CF and small bowel diseases. Gallstones are calcified in approximately $50 \%$, particularly if associated with haemolytic disorders.

US is the modality of choice for the evaluation of cholelithiasis. Gallstones will appear as echogenic foci with acoustic shadowing in the gallbladder or bile ducts (Fig. 12). In the gallbladder they often change position with gravity. Gallstones may cause biliary obstruction with dilatation of the bile ducts visualized on US (Fig. 13). They can spontaneously resolve. The differentiation of biliary sludge from small nonshadowing gallstones may be difficult. MRCP may play a role as a problem-solving technique in cases where US is inconclusive. Gallstones are visualized at MRCP as filling defects in the gallbladder or bile ducts (Fig. 14). If there is biliary obstruction MRCP clearly depicts the degree of biliary duct dilatation and level of obstruction.

\section{Cholangitis}

Biliary tract infections do occur in children and are often associated with congenital or immune-related bile duct abnormalities, surgically corrected biliary atresia (Kasai procedure) (Fig. 5), liver transplantation and certain immunodeficiency states [39, 40]. Infectious cholangitis usually has a bacterial origin, although viral, fungal and parasitic infections are also possible, especially in the immune-compromised host. The primary goal of the radiological evaluation in (suspected) infectious cholangitis is to search for evidence of anatomical abnormalities (e.g., choledochal cysts) and/or obstruction of the biliary tract. US is the initial imaging modality of choice in children followed by MRCP.

A distinct type of cholangitis, increasingly found in children, is primary sclerosing cholangitis (PSC) [41]. This is a chronic and usually progressive cholestatic liver disease of unknown origin, which can result in liver cirrhosis, 
Fig. 16 US and MRI in a 10month-old boy with liver cirrhosis due to a metabolic disease (tyrosinaemia). a-c US shows heterogeneous liver parenchyma with irregular contours (arrow in b) and a small hypoechoic regenerative nodule (c). $\mathbf{d}$ rt TSE T2-W image of the liver shows the heterogeneous liver parenchyma with irregular concaudate lobe (arrow). e Postgadolinium bh 3-D GRE T1-W image with fat suppression shows an almost homogeneous enhancement of the liver parenchyma without focal lesions tours and enlargement of the
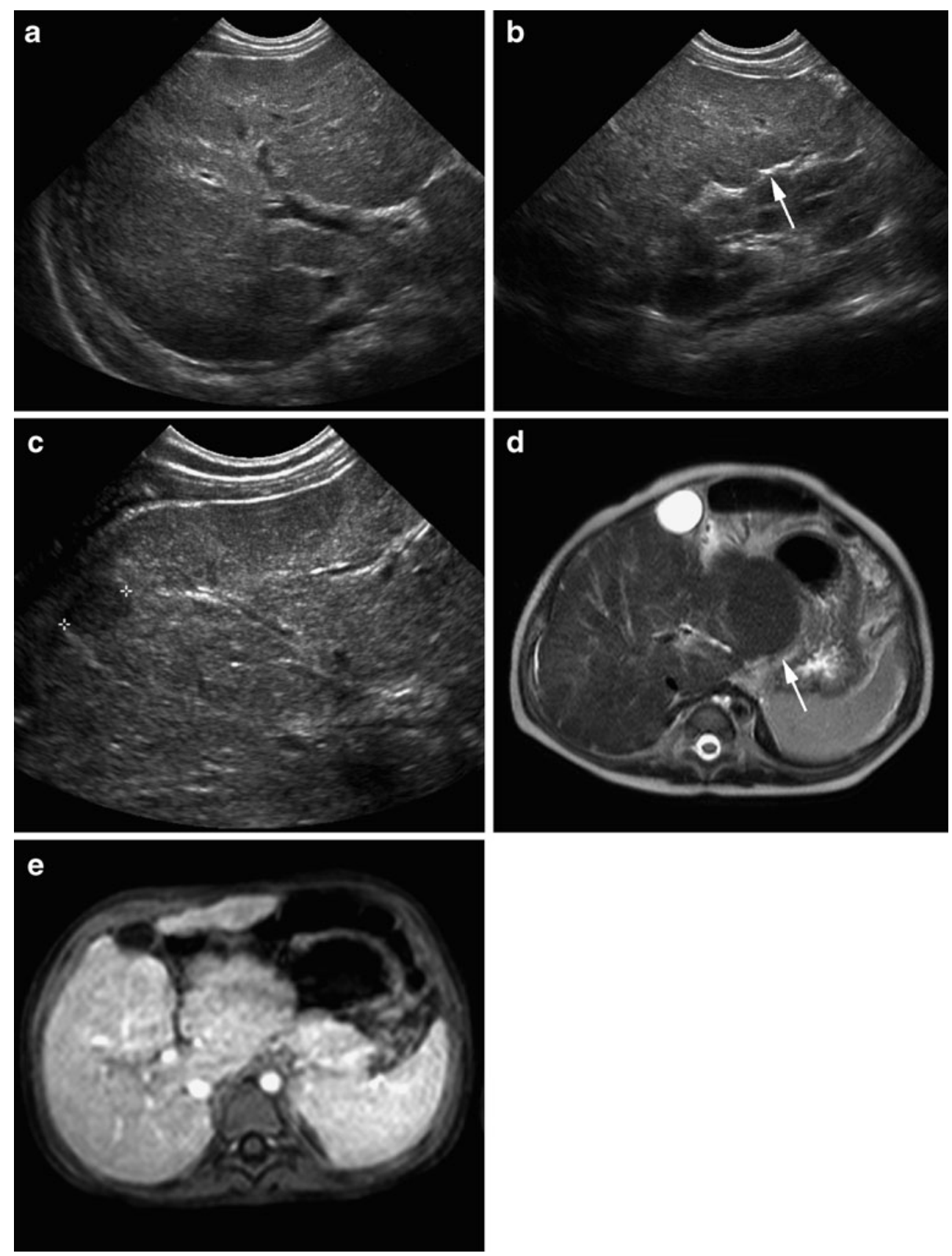

Fig 17 MRI in an 18-year-old boy with a more advanced stage of liver cirrhosis secondary to a mitochondrial disease. a rt TSE T1-W and (b) rt TSE T2-W axial images show marked heterogeneity of the liver parenchyma with multiple low T1signal intensity bands due to fibrotic stranding
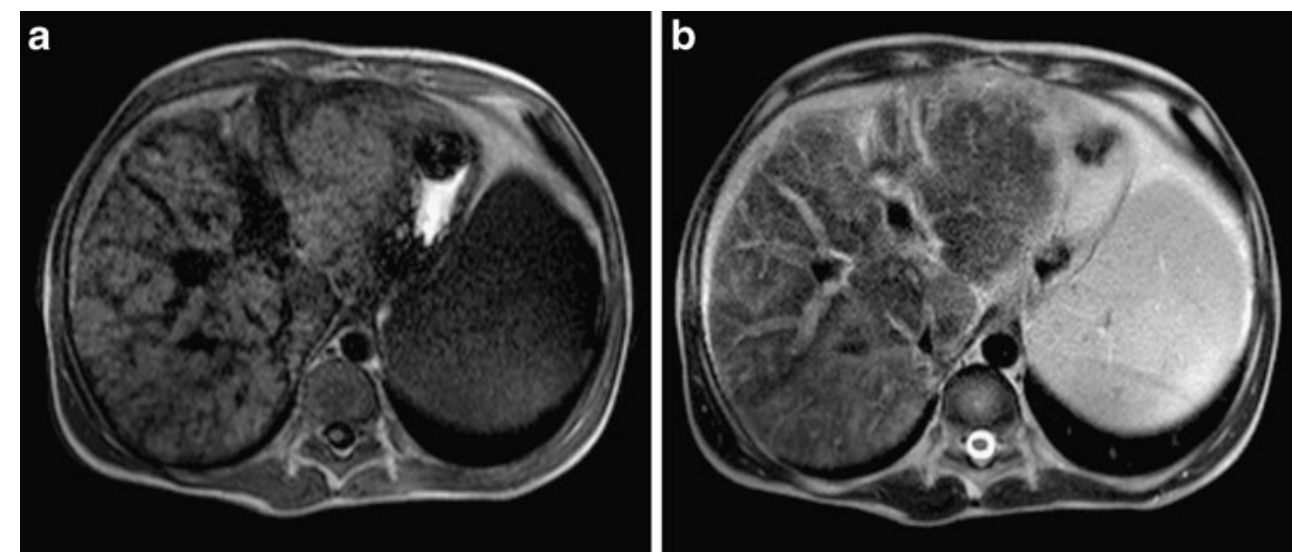
Fig. 18 US of the pancreatic head (a) and liver hilum (b) in a 9-year-old boy with a history of portal vein thrombosis. There are multiple collateral vessels visible in the region of the pancreatic head (arrowheads in a) and colour Doppler shows cavernous transformation of the portal vein (b)
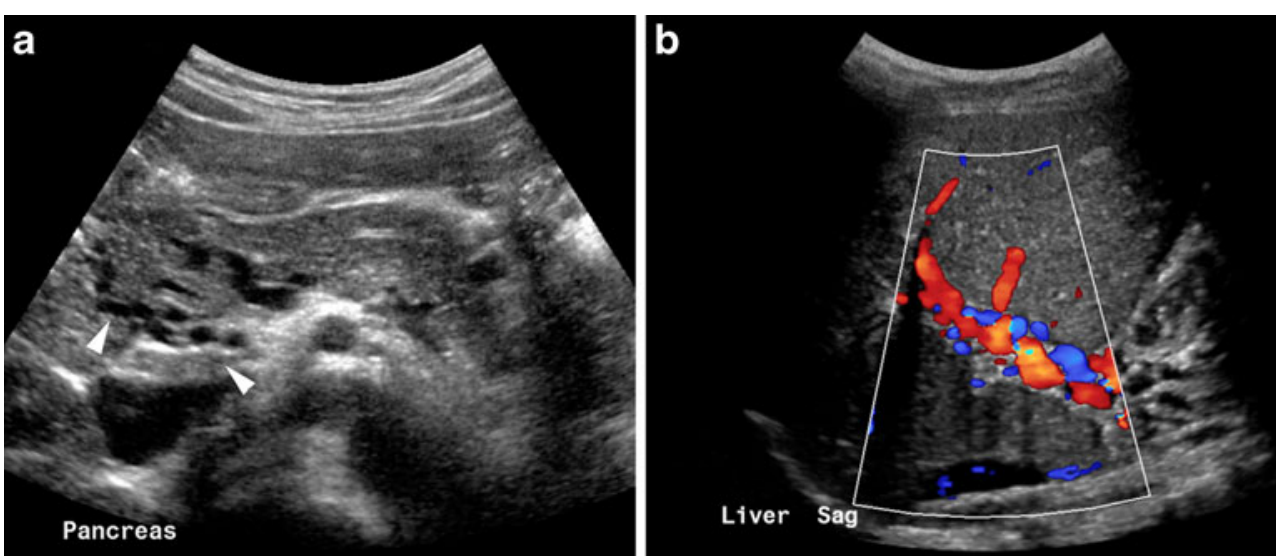

portal hypertension and liver failure. PSC is associated with inflammatory bowel diseases (IBD); $70 \%-80 \%$ of patients with PSC have IBD, and conversely, up to $7 \%$ of patients with IBD will develop PSC [42]. The diagnosis is based on clinical and typical imaging features, cholestatic biochemical profile and liver histology.

If PSC is suspected, MRCP is the initial modality of choice to evaluate the biliary system [11, 43]. The most characteristic findings are alternating dilatations and stenoses of the intra- and extrahepatic bile ducts together with peripheral wedge-shaped areas of high T2-signal intensities (Fig. 15). The overall diagnostic accuracy of MRCP in patients with PSC is reported to be around 90\%, compared to $97 \%$ for endoscopic retrograde cholangiopancreatography (ERCP). However, ERCP is an invasive technique with the risk of procedural complications. Furthermore, with ERCP it may be difficult or impossible to visualize the bile ducts proximal to the obstruction.

US plays a minor role in the evaluation of PSC. It may reveal intra- and/or extrahepatic bile duct dilatation. Furthermore, the liver parenchyma may show increased echogenicity and heterogeneity related to the cirrhosis. Splenomegaly and ascites can be seen in case of portal hypertension.
Fig. 19 US, CT and MRI in a 8.5-year-old girl presenting with acute abdominal pain and distension. a Colour Doppler US shows heterogeneous hyperechoic liver parenchyma with absent flow in the main hepatic veins of the right hepatic lobe (arrow), $L H V$ left hepatic vein. b Axial CT slice shows slightly heterogeneous enhancement and periportal oedema (arrowhead) as well as absent enhancement of the right hepatic veins (arrow). c and (d) rt TSE T2-W axial MR images show heterogeneous liver parenchyma, ascites, absent right hepatic veins (arrow in $\mathbf{c}$ ) and intrahepatic collaterals (open arrow in d), compatible with Budd-Chiari syndrome
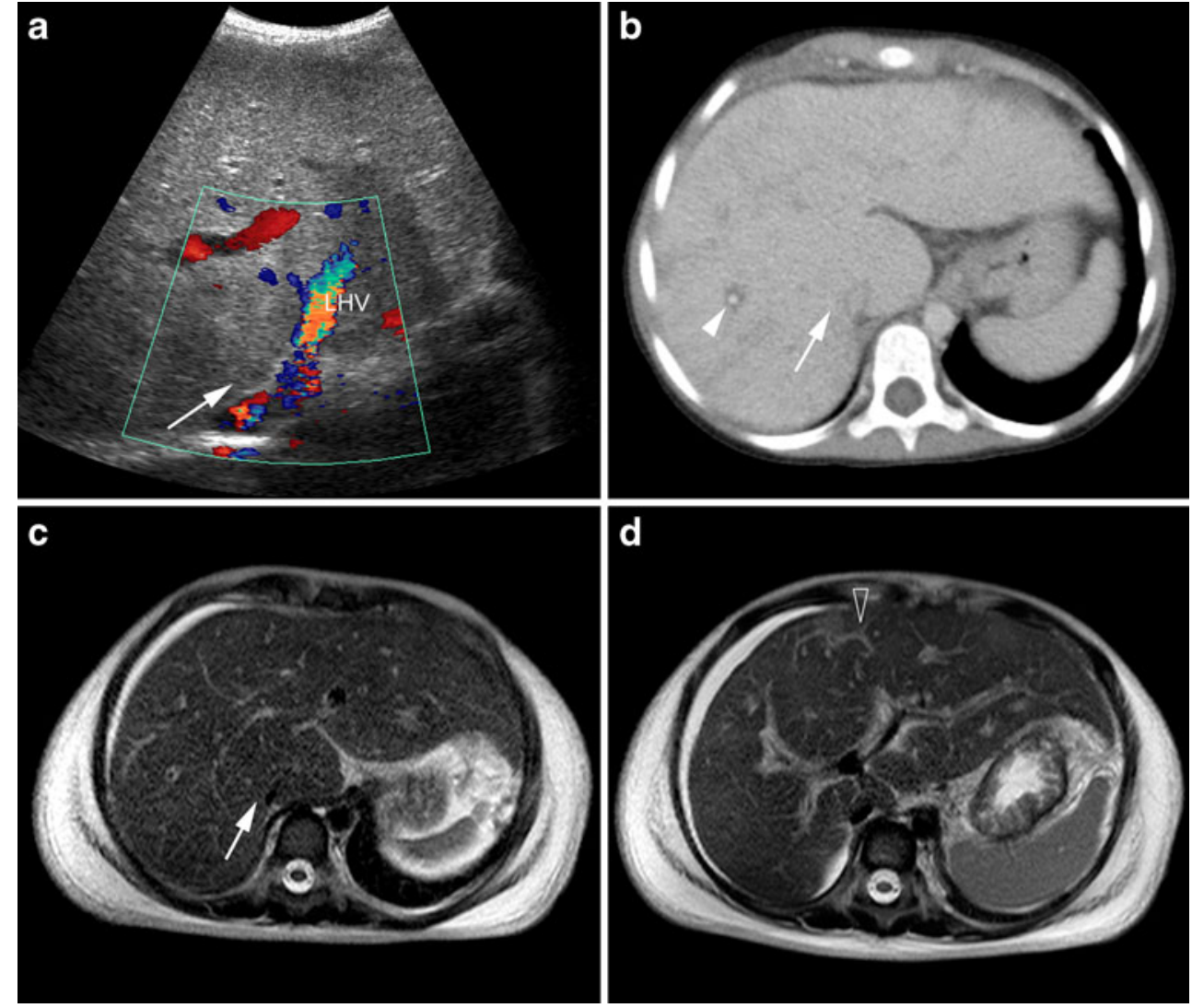


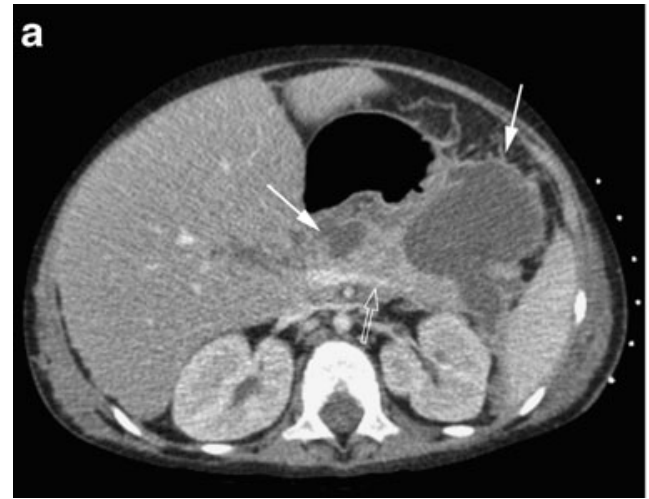

Fig. 20 Contrast-enhanced $\mathrm{CT}$ in a 3-year-old boy with acute lymphatic leukaemia and acute pancreatitis most likely related to Asparaginase (chemotherapy). The CT shows diffuse gland enlargement with mild inhomogeneous enhancement of the parenchyma (open arrow in a), irregular margins and inflammatory changes of the

\section{Diffuse liver disease}

\section{Cirrhosis}

Liver cirrhosis does occur in children and is usually secondary to a wide variety of congenital and acquired diseases, such as biliary atresia, CF, congenital hepatic fibrosis, metabolic disorders, hepatitis and Budd-Chiari syndrome [44]. It is characterized by widespread destruction of the liver parenchyma with fibrosis and regeneration in a focal nodular pattern (micro- and macronodules).

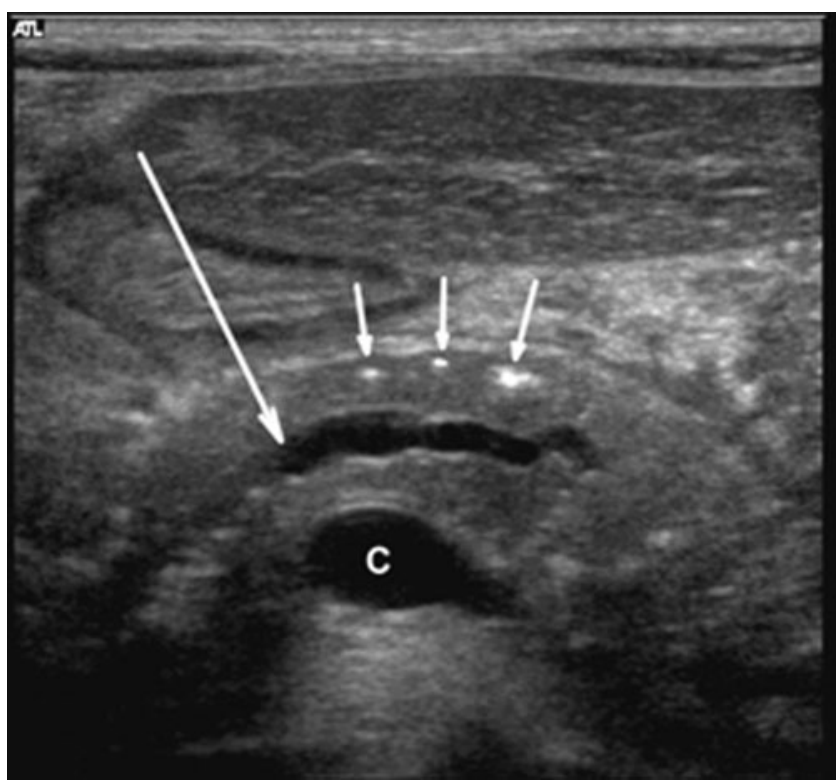

Fig. 21 Transverse US image in a 5-year-old boy with chronic hereditary pancreatitis shows the typical features of chronic pancreatitis: calcifications (small arrows) and dilatation of the pancreatic duct (large arrow). $C$, confluence of the superior mesenteric and splenic veins

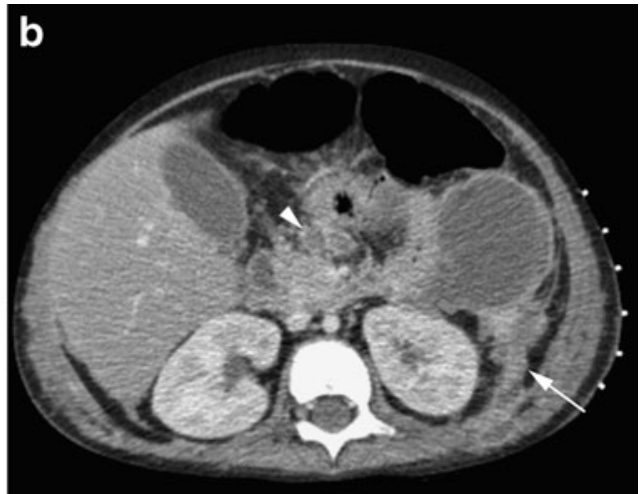

peripancreatic tissue (arrowhead in b), and multiple small and large peripancreatic fluid collections (arrows in a). The inflammation has spread anteriorly to the pararenal space with thickening of Gerotha's fascia and peritoneum (arrow in b)

Complications of cirrhosis are portal hypertension and hepatocellular carcinoma.

Again, US is the initial modality of choice for the diagnosis and follow-up of liver cirrhosis (Fig. 16) [3, 45, 46]. In the early phase the liver will appear normal-size or enlarged and heterogeneous with increased echogenicity due to the fibrosis and fatty infiltration. However, in more advanced cases, the right liver lobe will be small with irregular margins. The caudate lobe and lateral segment of the liver show compensatory enlargement. When regenerative nodules do occur, they usually have a relatively decreased echogenicity. The intrahepatic vessels may be small due to compression by fibrosis. Portal hypertension can develop with characteristic flow patterns in advanced stages (see below). Other signs are splenomegaly and ascites. In addition, as discussed in the imaging techniques

Table 6 Classification of blunt hepatic trauma [58]

\begin{tabular}{|c|c|}
\hline Grade & Criteria \\
\hline 1 & $\begin{array}{l}\text { Subcapsular haematoma }<10 \% \text { surface area, capsular } \\
\text { tear }<1 \mathrm{~cm} \text { parenchymal depth }\end{array}$ \\
\hline 2 & $\begin{array}{l}\text { Subcapsular haematoma } 10 \%-50 \% \text { surface area, } \\
\text { intraparenchymal haematoma }<10 \mathrm{~cm} \text { diameter, laceration } \\
1-3 \mathrm{~cm} \text { parenchymal depth and }<10 \mathrm{~cm} \text { length }\end{array}$ \\
\hline 3 & $\begin{array}{l}\text { Subcapsular haematoma }>50 \% \text { surface area or expanding, } \\
\text { ruptured subcapsular or parenchymal haematoma, } \\
\text { intraparenchymal haematoma }>10 \mathrm{~cm} \text { or expanding, } \\
\text { laceration }>3 \mathrm{~cm} \text { parenchymal depth }\end{array}$ \\
\hline 4 & $\begin{array}{l}\text { Parenchymal disruption involving } 25 \%-75 \% \text { of hepatic lobe } \\
\text { or } 1-3 \text { Couinaud's segments within a single lobe }\end{array}$ \\
\hline 5 & $\begin{array}{l}\text { Parenchymal disruption involving }>75 \% \text { of hepatic lobe } \\
\text { or }>3 \text { Couinaud's segments within a single lobe, } \\
\text { juxtahepatic venous injuries (retro hepatic vena cava, } \\
\text { central major hepatic veins) }\end{array}$ \\
\hline 6 & Hepatic avulsion \\
\hline
\end{tabular}


Fig. 22 US and CT of the liver in a 1.5-year-old girl with a liver laceration due to blunt abdominal trauma. a-c US shows a heterogeneous defect in the liver parenchyma (arrow in a) with anechoic parts (arrow in b) extending into the liver hilum (c). The sagittal (d) and coronal (e) MPR reconstructions of the contrast-enhanced CT better illustrate the full extent of the liver laceration (arrow in $\mathbf{d}$ and e) caudal in the right liver lobe reaching into the liver hilum but without evidence of major vascular injury (grade 4)
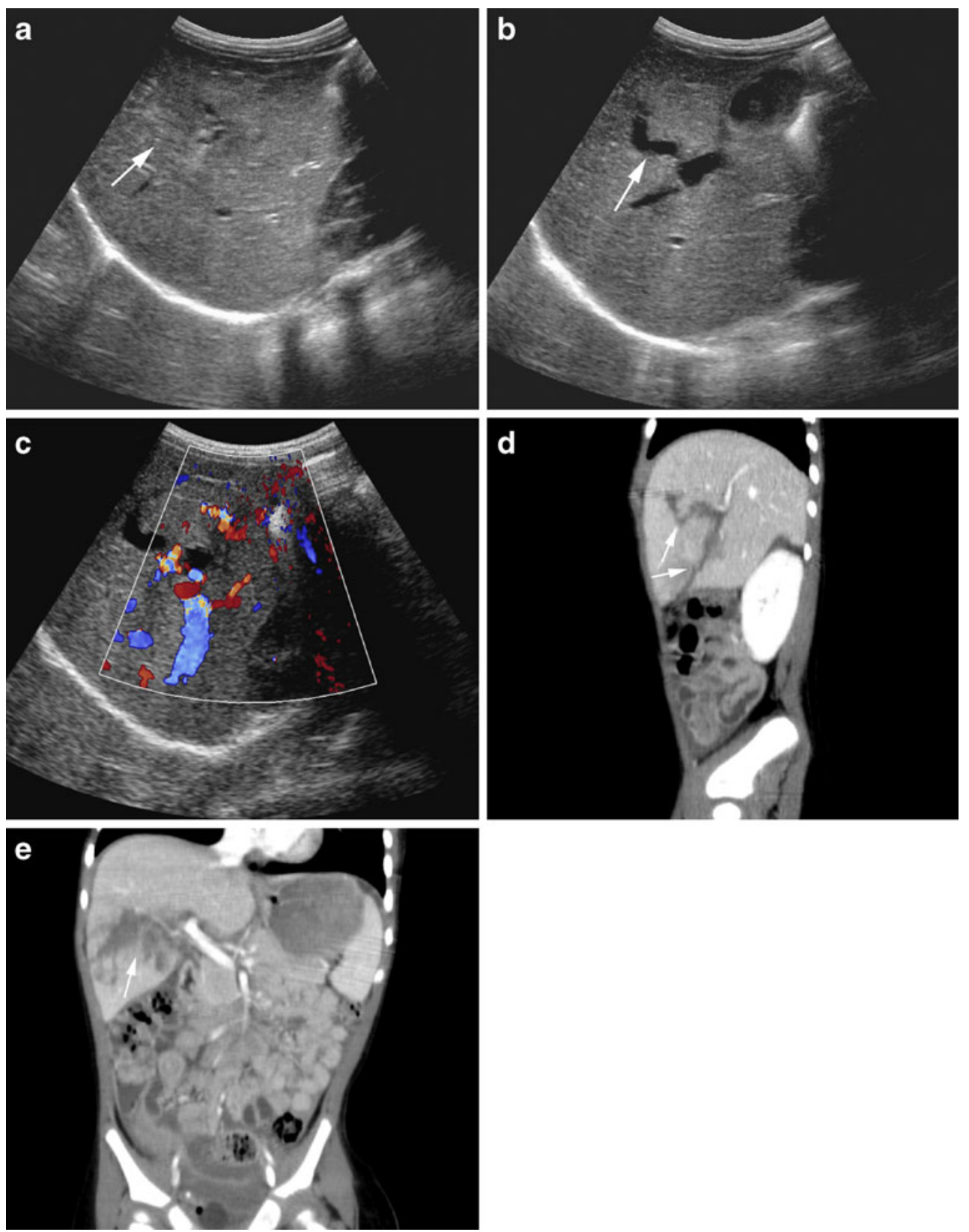

section, transient elastography may be a valuable noninvasive method to detect and quantify liver fibrosis and/or cirrhosis more accurately than grey-scale US alone [3-5].

MRI can play a role in advanced disease stages by demonstrating the full extent of the cirrhosis and its complications as well as in the differentiation of regenerating nodules from hepatocellular carcinoma [47, 48]. Characteristic morphological changes such as surface nodularity, widening of fissures, expansion of the gallbladder fossa, notching and/or atrophy of the right lobe, and relative enlargement of the left and caudate lobes are all well-visualized with MRI (Fig. 16) [47]. The signal intensity of cirrhosis is nonspecific and depends on the degree of fatty infiltration, fibrosis and iron deposition.
Fibrotic septa and bridges are usually of low-signal intensity on T1-W images and high-signal intensity on T2-W images (Fig. 17). The regenerative nodules typically have an intermediate- to high-signal intensity on $\mathrm{T} 1-\mathrm{W}$ images and intermediate- to low-signal intensity on $\mathrm{T} 2-\mathrm{W}$ images, although they may be markedly hypointense on T2W (especially GRE) images secondary to the iron deposition. In the latter, it can be difficult to differentiate them from hepatocellular carcinoma. Sometimes, nodules may be steatotic with signal loss on out-of-phase images. Contrastenhanced MR imaging (including the use of liver-specific contrast agents) can help to differentiate between regenerating nodules and HCC [48]. Regenerative nodules are usually relatively small $(<1 \mathrm{~cm})$ and have a similar arterial 
Fig. 23 Contrast-enhanced CT of the abdomen in a 12-year-old girl after blunt abdominal trauma. a Axial slice and (b) coronal MPR. There is free intraperitoneal fluid (asterisk in b) and a small liver laceration caudal in the right liver lobe with contrast medium extravasation due to active bleeding (arrow in $\mathbf{a}$ and $\mathbf{b}$ )
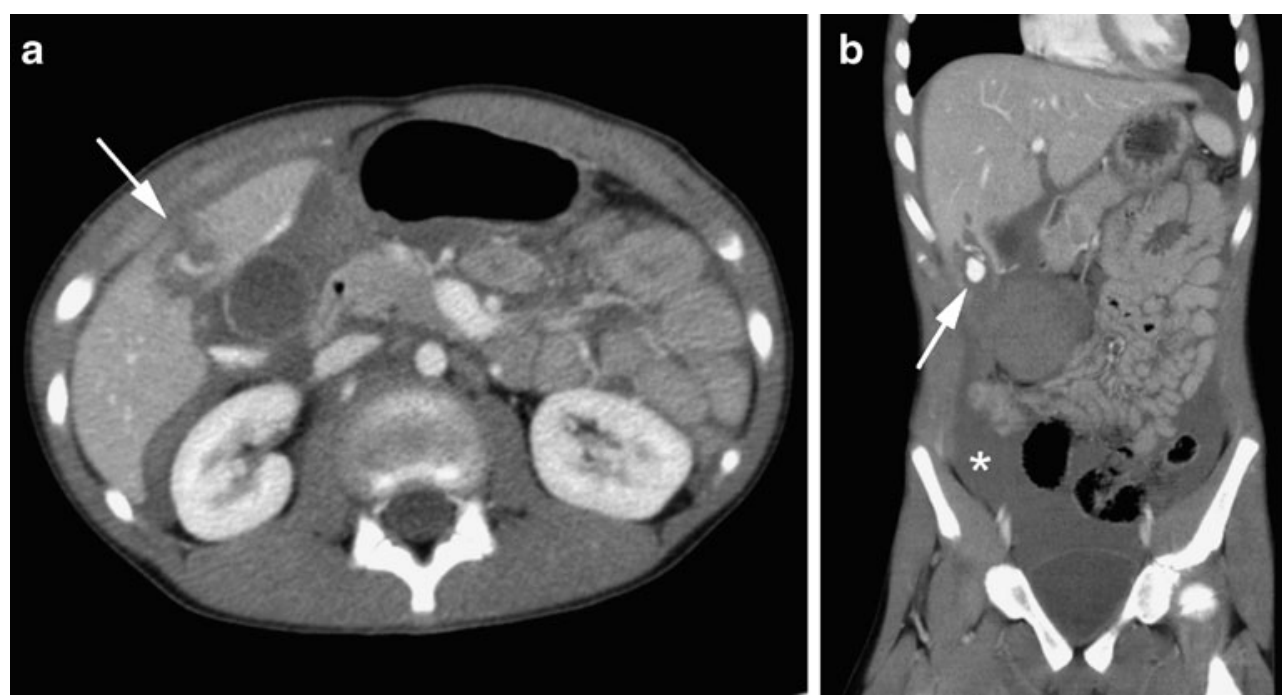

phase enhancement as the background liver parenchyma. On the contrary, HCC most typically shows intense enhancement during the arterial phase, is usually isointense during the portal venous phase and shows washout during the delayed phase. In addition, HCC most often has high-signal intensity on T2-W images. Lastly, regenerative nodules show a substantial uptake of iron oxide-based contrast agents, resulting in a signal decrease on contrast-enhanced T2-W images. The complications of liver cirrhosis, including portal hypertension with (splenorenal, splenocaval, etc.) shunt and collateral vascular development, ascites and splenomegaly are also easily revealed with MRI.

Although the above described characteristics of liver cirrhosis and its complications can also be demonstrated with $\mathrm{CT}, \mathrm{MRI}$ is the preferred advanced imaging modality in children because of the lack of ionizing radiation. When performed, CT will show a normal-size or small liver with irregular margins and areas of decreased attenuation due to fatty infiltration. Contrast enhancement is heterogeneous and signs of portal hypertension can be demonstrated with CT as well. Hepatic scintigraphy usually does not play a role in imaging cirrhosis.

\section{Portal hypertension}

In children, portal hypertension is usually due to extrahepatic portal obstruction, including idiopathic cavernous transformation, intrinsic portal vein webs, portal vein thrombosis related to umbilical vein catheterization, postoperative complications and masses in the porta hepatis [44]. It remains clinically silent until either splenomegaly is detected or upper GI bleeding occurs. The most common intrahepatic cause of portal hypertension is liver cirrhosis.

On US, splenomegaly and ascites can be detected, the latter being more common in the intrahepatic form of portal hypertension. In patients with extrahepatic portal obstruction, the portal vein will be small or cannot be identified, and serpentine, tortuous collaterals can be seen in the porta hepatis (cavernous transformation) (Fig. 18). Collaterals can be identified in the ligamentum teres (paraumbilical collateral), splenic hilum, gastrohepatic ligament and adjacent to the oesophagogastric junction. Intrahepatic causes of portal hypertension will show an increase in portal vein diameter, exceeding $13 \mathrm{~mm}$, decrease in flow velocity $(<15-18 \mathrm{~cm} / \mathrm{s})$ and loss of normal respiratory pulsations [3]. With further progression of the intrahepatic parenchymal changes the flow in the portal vein may disappear or become reversed (hepatofugal) flow and portal vein diameter may decrease, whereas the hepatic artery commonly becomes more prominent. However, in case of extensive collateral formation these signs may be less well-established and the portal vein flow may remain hepatopetal. Therefore, these Doppler signs have proved to be unreliable in predicting the degree of liver fibrosis/cirrhosis [3].

MRI and MR angiography (MRA) can be used to evaluate the portal venous system [47]. Usually, standard T1- and T2$\mathrm{W}$ imaging of the liver parenchyma will precede the postgadolinium imaging and MRA of the hepatic vessels. In precontrast T1-W imaging the hepatic vessels will appear black, whereas they will be bright on contrast-enhanced T1-W imaging and MRA. Thrombosis of the portal vein can be identified as an intravascular filling defect on both pre- and post-contrast imaging, although this can be simulated by slow or stagnant flow. Identification of the varices and collateral pathways is usually possible. Although CT can demonstrate these issues with similar accuracy, MRI is again preferred because of the lack of ionizing radiation.

\section{Budd-Chiari syndrome}

This is a rare entity in children caused by a hepatic venous obstruction. It is often idiopathic, but can be associated with 

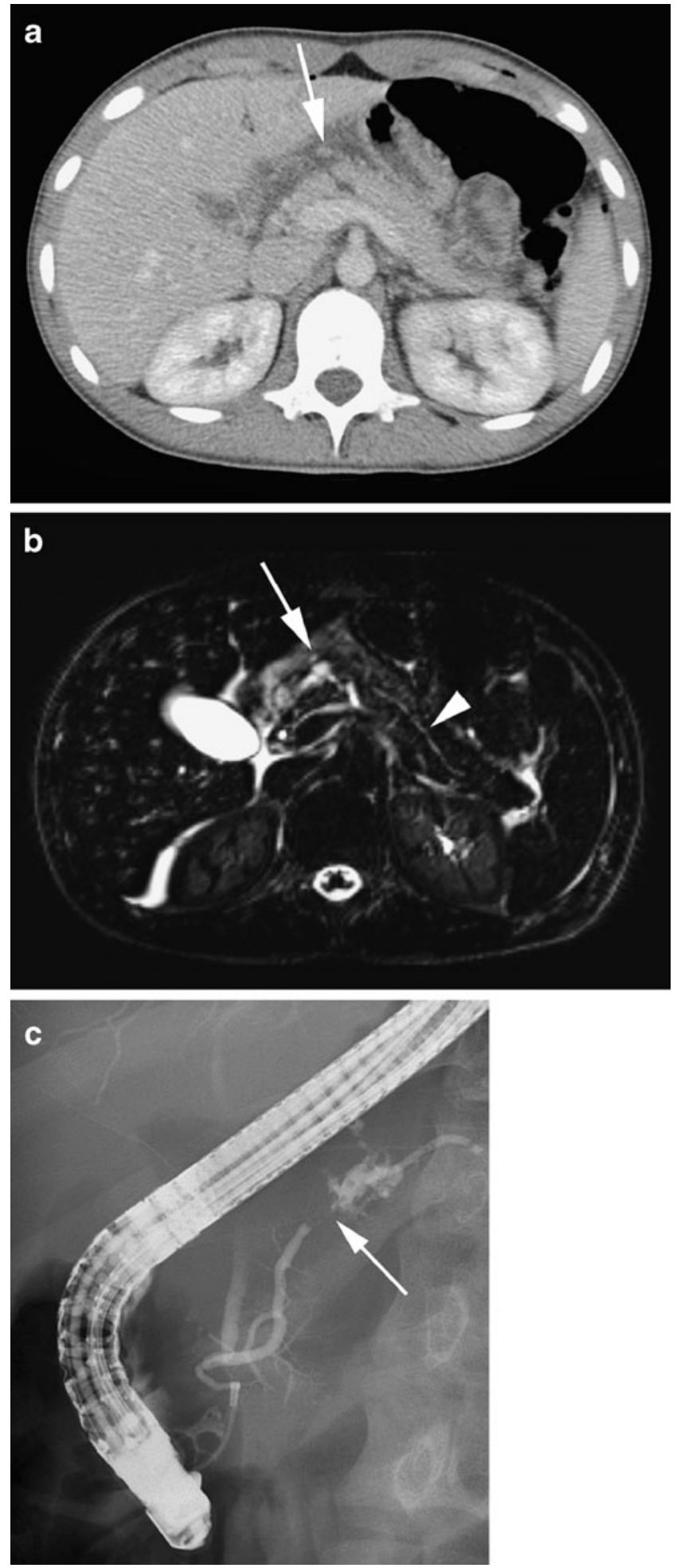

Fig. 24 CT, MRI and ERCP in a 14-year-old girl after a bicycle accident (steering-column injury). a Axial contrast-enhanced CT slice and (b) axial T2-W TSE MR image show laceration of the corpus of the pancreas (arrow) with a local retroperitoneal fluid collection, due to haematoma and leakage of pancreatic fluid. The axial MR image shows more clearly the concomitant transection of the pancreatic duct (arrowhead). c ERCP confirms transection of the pancreatic duct (arrow) with extravasation of contrast material (courtesy of R.R. van Rijn, AMC, Amsterdam, The Netherlands) hypercoagulable states, congenital venous webs (intrahepatic or caval), neoplasms, trauma and abdominal surgery [44]. Children with Budd-Chiari syndrome usually present with rapid-onset abdominal distension and pain due to hepatomegaly and ascites.

On US the liver may appear heterogeneous with focal hypoechoic areas secondary to haemorrhagic infarction or necrosis (Fig. 19) [49, 50]. Later on, fibrosis and scarring may result in hyperechoic regions. The liver is usually enlarged, especially the caudate lobe, and ascites may be seen. The hepatic veins can be partly or completely occluded and US is helpful to identify the level of obstruction (Fig. 19). Markedly reduced or reversed flow in the hepatic veins and/or inferior vena cava can be seen on Doppler evaluation.

CT and MRI are both accurate but not highly specific in identifying Budd-Chiari syndrome [50]. Typical features include hepatomegaly with an enlarged caudate lobe, heterogeneous liver parenchyma, absent or narrowed hepatic veins, thrombus in the hepatic veins, intrahepatic venous collaterals, thrombosis or compression of the inferior vena cava and ascites (Fig. 19). MRA may demonstrate hepatic venous obstruction and collateral circulation, probably as accurately as Doppler US and angiography.

\section{Pancreatitis}

Acute and chronic inflammatory diseases of the pancreas in childhood cause significant morbidity and mortality [51, 52]. Acute pancreatitis is idiopathic in $23 \%$ of patients. Other aetiologies are trauma $(22 \%)$, structural anomalies (15\%), multisystem diseases (14\%), drugs and toxins (12\%) and viral infections $(10 \%)$. US is the primary imaging modality for detection of pancreatic abnormalities and for exclusion of extrapancreatic disease [53]. CT is widely used for further evaluation and MRI (including MRCP) is emerging as the modality of choice. ERCP is becoming an interventional modality. The aetiology of chronic pancreatitis includes $\mathrm{CF}$, fibrosing pancreatitis, hereditary chronic pancreatitis and inborn errors of metabolism.

In case of acute pancreatitis, the echogenicity of the pancreas on US is not a helpful diagnostic feature; pancreatic enlargement is absent in $50 \%$ of the patients. The most useful diagnostic feature is dilatation of the pancreatic duct $(>1.5 \mathrm{~mm}$ at $1-6$ years of age, $>1.9 \mathrm{~mm}$ at 7-12 years and $>2.2 \mathrm{~mm}$ at $13-18$ years) [54]. On CT, the imaging features include swelling of the pancreas, blurring of the contours, inhomogeneous enhancement, inflammatory changes in the peripancreatic fat, intra- or retroperitoneal fluid collections and thickening of facial planes (Fig. 20). MRI with pre- and postcontrast sequences, before and after 
Table 7 Classification of pancreatic trauma [66]

\begin{tabular}{ll}
\hline Grade & Criteria \\
\hline 1 & Minor contusion or superficial laceration without duct injury \\
2 & Major contusion or laceration without duct injury or tissue loss \\
3 & Distal transection or parenchymal injury with duct injury \\
4 & $\begin{array}{l}\text { Proximal (right of the superior mesenteric vein) transection } \\
\text { or parenchymal injury involving the ampulla }\end{array}$ \\
5 & Massive disruption of the pancreatic head
\end{tabular}

secretin administration, including MRCP is shown to be a reliable alternative to contrast-enhanced $\mathrm{CT}$ in adults and may emerge as the next possible imaging technique after US [53]. In chronic pancreatitis, imaging features (both on US and CT) include tissue loss, irregular contours, ductal dilatation and ductal and parenchymal calcifications (Fig. 21).

\section{Trauma}

\section{Liver}

In case of blunt abdominal trauma, the liver is a commonly injured organ, especially in children because their ribs are more flexible [55-57]. The vast majority of blunt liver injuries in children are due to bicycle and pedestrian accidents. The most common mechanism is a child being struck by a car while cycling or crossing the road, although steering-column injuries also play a major role. In the neonate, a liver injury may be caused by a traumatic delivery or resuscitative effort. Liver injuries usually involve segments 6,7 and 8, and may range from a minor tear of the capsule to a full-blown laceration with injury of the vascular supply and/or bile ducts of the liver. In more than $50 \%$ of patients the liver injury is associated with other upper abdominal injuries, especially injuries to the spleen. Currently, most blunt liver injuries are treated conservatively (up to $97 \%$ in children), and surgery or interventional radiology is only indicated in the haemodynamically unstable child or when conservative treatment fails.

Imaging plays a vital role in grading the severity of the liver injury and during follow-up. Liver injuries are usually graded according to the American Association for the Surgery of Trauma (AAST) CT-based classification system into six severity grades (Table 6) [58]. US may play a role as a screening technique in the acute phase at the emergency department $[59,60]$. It is particularly useful in the neonate suspected of having a liver injury. In cases where US shows large amounts of intra-abdominal fluid, the patient will be immediately transported for surgery when unstable, whereas the stable patient may be imaged with CT to depict the full extent of intra-abdominal injury. US can demonstrate traumatic liver injuries (Fig. 22). A subcapsular haematoma initially will be anechoic, becoming progressively echogenic in the first $24 \mathrm{~h}$. In the next days, the haematoma once again becomes hypo- or anechoic, and in the chronic phase septa and internal echoes may appear. Parenchymal contusions will show a similar pattern over time. Lacerations of the liver parenchyma are usually slightly echogenic in the acute phase, becoming hypoechogenic or cystic when scanned several days later. The sensitivity of US increases with increasing severity of liver injury, being approximately $98 \%$ in injuries of grade 3 and higher. However, a negative US does not exclude liver injury. On the other hand, it is the ideal imaging modality for the follow-up of detected liver lesions.

MDCT (with intravenous contrast medium) is still the imaging modality of choice in the acute phase in patients with blunt abdominal trauma [56, 57, 61]. It is accurate in demonstrating site and extent of liver injury as well as other intra-abdominal traumatic lesions, providing important information for the treatment of the patient. MDCT has the advantage of fast scanning times, allowing scanning in specific post-contrast phases, especially when an active bleeding site is suspected (Fig. 23). However, some publications suggest that a monophasic protocol also will be adequate, which is preferred in children because of the risks of ionizing radiation. Subcapsular haematomas may appear as low-attenuating, lenticular collections between the liver capsule and enhancing liver parenchyma, with direct compression and deformity of the liver shape. Acute intraparenchymal haematomas are usually irregularly shaped, high-attenuation areas on contrast-enhanced CT, surrounded by low-attenuating unclotted blood or bile. During resorption of the haematoma the attenuation will reduce, eventually followed by formation of a defined serous fluid collection. A focal hyperattenuating area of 80 $350 \mathrm{HU}$ is believed to represent active haemorrhage or pseudoaneurysm. Lacerations often appear as nonenhancing linear or branching structures, usually in the periphery of the liver (Fig. 22). They may communicate with hepatic vessels and/or bile ducts (Fig. 23). Bilomas usually are diagnosed during follow-up, as they take weeks or months to develop because of the slow rate of leaking. They are usually low attenuating cystic structures in or around the liver.

The role of MRI in the diagnosis and follow-up of liver injuries still has to be established. In the acute phase, the role of MRI will be limited, but in the follow-up MRI can be used for monitoring the liver lesions, especially in children in whom the radiation dose of CT is a concern. MRCP can be used to demonstrate bile duct lesions [62]. Scintigraphy with ${ }^{99 \mathrm{~m}}$ Tc IDA can be used to evaluate bile leaks. 


\section{Pancreas}

The pancreas is vulnerable to blunt abdominal trauma because of its fixed retroperitoneal position. In children with blunt abdominal trauma, pancreatic damage occurs in $3-12 \%$ [63]. Bicycle injuries are common and child abuse may result in pancreatic injuries in infants.

In general, posttraumatic pancreatic injury appears similar to that in other solid organs: oedema, haematoma, laceration or fracture [64]. These last three injuries have decreased echogenicity on US and low attenuation at CT and do not enhance after IV contrast administration (Fig. 24). One should consider that a normal CT performed shortly after trauma may be a false-negative examination because oedema and peripancreatic fluid develop only after several hours in minor to moderate trauma. MRCP seems to be the best modality to evaluate ductal laceration (Fig. 24) [64, 65].

Pancreatic injury is graded from 1 to 5 according to the Organ Injury Scaling of the American Association for the Surgery of Trauma (Table 7) [66]. In a series of 34 children, nonoperative management had a good clinical outcome and secondary surgery was necessary in only $10 \%$ [63]. Pseudocysts develop in $50 \%$ of the patients who are treated conservatively, of whom half can be managed nonoperatively [64].

\section{Conclusion}

As illustrated in this review, imaging plays a major role in the diagnostic work-up of children with hepatobiliary or pancreatic diseases. US is almost always the first imaging modality of choice, followed by MRI. CT can be reserved for imaging trauma and staging of malignancies, although for the latter MRI is increasingly validated and used.

Open Access This article is distributed under the terms of the Creative Commons Attribution Noncommercial License which permits any noncommercial use, distribution, and reproduction in any medium, provided the original author(s) and source are credited.

\section{References}

1. Konus OL, Ozdemir A, Akkaya A et al (1998) Normal liver, spleen, and kidney dimensions in neonates, infants, and children: evaluation with sonography. AJR 171:1693-1698

2. Siegel MJ, Martin KW, Worthington JL (1987) Normal and abnormal pancreas in children: US studies. Radiology 165:15-18

3. Goyal N, Jain N, Rachapalli V et al (2009) Non-invasive evaluation of liver cirrhosis using ultrasound. Clin Radiol 64:1056-1066

4. Fraquelli M, Rigamonti C, Casazza G et al (2007) Reproducibility of transient elastography in the evaluation of liver fibrosis in patients with chronic liver disease. Gut 56:968-973
5. Menten R, Leonard A, Clapuyt P et al (2010) Transient elastography in patients with cystic fibrosis. Pediatr Radiol 40:1231-1235

6. Nievelstein RA, Van Dam I, Van Der Molen AJ (2010) Multidetector $\mathrm{CT}$ in children: current concepts and dose reduction strategies. Pediatr Radiol 40:1324-1344

7. Riccabona M (2008) Potential of MR-imaging in the paediatric abdomen. Eur J Radiol 68:235-244

8. Schneider G, Grazioli L, Saini S eds (2006) Chapters 1 and 10. In: MRI of the liver, 2nd edn. Springer, Berlin

9. Albuquerque PA, Morales Ramos DA, Faingold R (2009) Magnetic resonance imaging of the liver and biliary tree. Curr Probl Diagn Radiol 38:126-134

10. Fitoz S, Erden A, Boruban S (2007) Magnetic resonance cholangiopancreaticography of biliary system abnormalities in children. Clin Imaging 31:93-101

11. Chavhan GB, Babyn PS, Manson D et al (2008) Pediatric MR cholangiopancreatography: principles, technique, and clinical applications. Radiographics 28:1951-62

12. Delaney L, Applegate KE, Karmazyn B et al (2008) MR cholangiopancreatography in children: feasibility, safety, and initial experience. Pediatr Radiol 38:64-75

13. Bates MD, Bucuvales JC, Alonso MH et al (1998) Biliary atresia: pathogenesis and treatment. Semin Liver Dis 18:281-293

14. Kelly DA, Davenport M (2007) Current management of biliary atresia. Arch Dis Child 92:1132-1135

15. Lowe LH, Schlesinger AE (2008) Congenital abnormalities (chapter 112). In: Slovis T (ed) Caffey's Pediatric diagnostic imaging, 11th edn, Section VI: the abdomen, pelvis and retroperitoneum. Part IV: hepatobiliary system. Mosby Elsevier, Amsterdam

16. Kanegawa K, Akasaka Y, Kitamura E et al (2003) Sonographic diagnosis of biliary atresia in pediatric patients using the "triangular cord" sign versus gallbladder length and contraction. AJR 181:1387-1390

17. Humphrey TM, Stringer MD (2007) Biliary atresia: US diagnosis. Radiology 244:845-851

18. Lee MS, Kim MJ, Lee MJ et al (2009) Biliary atresia: color doppler US findings in neonates and infants. Radiology 252:282289

19. Sevilla A, Howman-Gile R, Saleh H et al (2007) Hepatobiliary scintigraphy with SPECT in infancy. Clin Nucl Med 32:16-23

20. Yang JG, Ma DQ, Peng Y et al (2009) Comparison of different diagnostic methods for differentiating biliary atresia from idiopathic neonatal hepatitis. Clin Imaging 33:439-46

21. Arora NK, Kohli R, Gupta DK et al (2001) Hepatic technetium99m-mebrofenin iminodiacetate scans and serum gammaglutamyl transpeptidase levels interpreted in series to differentiate between extrahepatic biliary atresia and neonatal hepatitis. Acta Paediatr 90:975-981

22. Stringer MD, Dhawan A, Davenport M et al (1995) Choledochal cysts: lessons from a 20 year experience. Arch Dis Child 73:528531

23. Miyano T, Yamataka A (1997) Choledochal cysts. Curr Opin Pediatr 9:283-288

24. Todani T, Watanabe Y, Narusue M et al (1977) Congenital bile duct cysts: Classification, operative procedures, and review of thirty-seven cases including cancer arising from choledochal cyst. Am J Surg 134:263-269

25. De Vries JS, De Vries S, Aronson DC et al (2002) Choledochal cysts: age of presentation, symptoms, and late complications related to Todani's classification. J Pediatr Surg 11:1568-1573

26. Lowe LH (2008) Imaging hepatobiliary disease in children. Semin Roentgenol 43:39-49

27. Metreweli C, So NMC, Chu WCW et al (2004) Magnetic resonance cholangiography in children. Br J Radiol 77:1059-1064 
28. Kaste SC (2008) The pancreas (chapter 120). In: Slovis TL (ed) Caffey's Pediatric diagnostic imaging, 11th edn, Section VI: the abdomen, pelvis and retroperitoneum. Part VI: the pancreas. Mosby Elsevier, Amsterdam

29. Mogayzel PJ Jr, Flume PA (2010) Update in cystic fibrosis 2009. Am J Respir Crit Care Med 181:539-544

30. Colombo C (2007) Liver disease in cystic fibrosis. Curr Opin Pulm Med 13:529-536

31. Akata D, Akhan O (2007) Liver manifestations of cystic fibrosis. Eur J Radiol 61:11-17

32. Chaudry G, Navarro OM, Levine DS et al (2006) Abdominal manifestations of cystic fibrosis in children. Pediatr Radiol $36: 233-240$

33. Akata D, Akhan O, Ozcelik U et al (2002) Hepatobiliary manifestations of cystic fibrosis in children: correlation of CT and US findings. Eur J Radiol 41:26-33

34. Mueller-Abt PR, Frawly KJ, Greer RM et al (2008) Comparison of ultrasound and biopsy findings in children with cystic fibrosis related liver disease. J Cyst Fibros 7:215-221

35. Taylor CJ, Aswani N (2002) The pancreas in cystic fibrosis. Paediatr Respir Rev 3:77-81

36. Cohen MM (2005) Beckwith-Wiedemann syndrome: historical, clinicopathological, and etiopathogenetic perspectives. Pediatr Dev Pathol 8:287-304

37. Spivey PS, Bradshaw WT (2009) Recognition and management of the infant with Beckwith-Wiedemann syndrome. Adv Neonatal Care 9:279-284

38. Wesdorp I, Bosman D, De Graaff A et al (2000) Clinical presentation and predisposing factors of cholelithiasis and sludge in children. J Pediatr Gastroenterol Nutr 31:411-417

39. Lowe LH, Schlesinger AE (2008) Acquired biliary tract disease (chapter 116). In: Slovis TL (ed) Caffey's Pediatric diagnostic imaging, 11th edn. Section VI: the abdomen, pelvis and retroperitoneum. Part IV: hepatobiliary system. Mosby Elsevier, Amsterdam

40. Yasuda H, Takada T, Kawarada Y et al (2007) Unusual causes of acute cholecystitis and cholangitis: Tokyo guidelines. J Hepatobiliary Pancreat Surg 14:98-113

41. Kaplan GG, Laupland KB, Butzner D et al (2007) The burden of large and small duct primary sclerosing cholangitis in adults and children: a population-based analysis. Am J Gastroenterol 102:1042-1049

42. Danese S, Semeraro S, Papa A et al (2005) Extraintestinal manifestations in inflammatory bowel disease. World J Gastroenterol 11:7227-7236

43. Chavhan GB, Roberts E, Moineddin R et al (2008) Primary sclerosing cholangitis in children: utility of magnetic resonance cholangiopancreatography. Pediatr Radiol 38:868-873

44. Lowe LH (2008) Diffuse parenchymal disease (chapter 114). In: Slovis TL (ed) Caffey's Pediatric diagnostic imaging, 11th edn. Section VI: the abdomen, pelvis and retroperitoneum. Part IV: hepatobiliary system. Mosby Elsevier, Amsterdam

45. Zheng RQ, Wang QH, Lu MD et al (2003) Liver fibrosis in chronic viral hepatitis: an ultrasonographic study. World J Gastroenterol 9:2484-2489
46. Zhu JA, Hu B (2003) Ultrasonography in predicting and screening liver cirrhosis in children: a preliminary study. World J Gastroenterol 9:2348-2349

47. Faria SC, Ganesan K, Mwangi I et al (2009) MR imaging of liver fibrosis: current state of the art. Radiographics 29:1615-1635

48. Hussain SM, Reinhold C, Mitchell DG (2009) Cirrhosis and lesion characterization at MR imaging. Radiographics 29:16371652

49. Chaubal N, Dighe M, Hanchate V et al (2006) Sonography in Budd-Chiari syndrome. J Ultrasound Med 25:373-379

50. Cura M, Haskal Z, Lopera J (2009) Diagnostic and interventional radiology for Budd-Chiari syndrome. Radiographics 29:669-681

51. Benifla M, Weizman Z (2003) Acute pancreatitis in childhood: analysis of literature data. J Clin Gastroenterol 37:169-172

52. Nydegger A, Couper RT, Oliver MR (2006) Childhood pancreatitis. J Gastroenterol Hepatol 21:499-509

53. Darge K, Anupindi S (2009) Pancreatitis and the role of US, MRCP and ERCP. Pediatr Radiol 39:S153-S157

54. Chao HC, Lin SJ, Kong MS et al (2000) Sonographic evaluation of the pancreatic duct in normal children and children with pancreatitis. J Ultrasound Med 19:757-763

55. Gaines BA, Ford HR (2002) Abdominal and pelvic trauma in children. Crit Care Med 30:S416-S423

56. Wegner S, Coletti JE, Van Wie D (2006) Pediatric blunt abdominal trauma. Pediatr Clin North Am 53:243-256

57. Sivit CJ (2009) Imaging children with abdominal trauma. AJR 192:1179-1189

58. Moore EE, Cogbill TH, Jurkovich GJ et al (1995) Organ injury scaling: spleen and liver (1994 revision). J Trauma 38:323-324

59. Nural MS, Yardan T, Güven H et al (2005) Diagnostic value of ultrasonography in the evaluation of blunt abdominal trauma. Diagn Interv Radiol 11:41-44

60. Holmes JF, Gladman A, Chang CH (2007) Performance of abdominal ultrasonography in pediatric blunt trauma patients: a meta-analysis. J Pediatr Surg 42:1588-1594

61. Browning JG, Wilkinson AG, Beattie T (2008) Imaging paediatric blunt abdominal trauma in the emergency department: ultrasound versus computed tomography. Emerg Med J 25:645-648

62. Kelly MD, Armstrong CP, Longstaff A (2008) Characterization of biliary injury from blunt liver trauma by MRCP: case report. J Trauma 64:1363-1365

63. Shilyansky J, Sena LM, Kreller M et al (1998) Nonoperative management of pancreatic injuries in children. J Pediatr Surg 33:343-349

64. Bosboom D, Braam AW, Blickman JG et al (2006) The role of imaging studies in pancreatic injury due to blunt abdominal trauma in children. Eur J Radiol 59:3-7

65. Gillams AR, Kurzawinski T, Lees WR (2006) Diagnosis of duct disruption and assessment of pancreatic leak with dynamic secretin-stimulated MR cholangiopancreatography. AJR 186:499-506

66. Moore EE, Cogbill TH, Malangoni MA et al (1990) Organ injury scaling, II: pancreas, duodenum, small bowel, colon, and rectum. J Trauma 30:1427-1429 